\title{
Nuclear Factor E2-Related Factor-2 Negatively Regulates NLRP3 Inflammasome Activity by Inhibiting Reactive Oxygen Species-Induced NLRP3 Priming
}

\author{
Xiuting Liu, Xin Zhang, Yang Ding, Wei Zhou, Lei Tao, Ping Lu, Yajing Wang, and Rong Hu
}

\begin{abstract}
Aims: The NLRP3 inflammasome is a multiprotein complex that protects hosts against a variety of pathogens. However, the molecular mechanisms of modulating NLRP3 inflammasome activation, especially at the priming step, are still poorly understood. This study was designed to elucidate the negative regulation of nuclear factor E2-related factor-2 (Nrf2) on the activation of NLRP3 inflammasome.

Results: We reported that Nrf2 activation inhibited NLRP3 expression, caspase-1 cleavage, and subsequent IL-1 $\beta$ generation. Compared with normal cells, Nrf2-deficient cells showed upregulated cleaved caspase-1, which were attributed to the increased transcription of NLRP3 caused by excess reactive oxygen species (ROS). Furthermore, priming of the NLRP3 inflammasome was sensitive to the exogenous ROS levels induced by $\mathrm{H}_{2} \mathrm{O}_{2}$ or rotenone. Combined with adenosine triphosphate, rotenone triggered higher activity of the NLRP3 inflammasome compared with lipopolysaccharide, suggesting that ROS promoted the priming step. In addition, Nrf2-induced NQO1 was involved in the inhibition of the NLRP3 inflammasome. In an in vivo alum-induced peritonitis mouse model, Nrf2 activation suppressed typical IL-1 signaling-dependent inflammation, whereas $\mathrm{Nrf} 2^{-1-}$ mice exhibited a significant increase in the recruitment of immune cell and the generation of IL-1 $\beta$ compared with wild-type mice.

Innovation: We elucidated the effects and possible mechanisms of Nrf2 activation-induced NQO1 expression on NLRP3 inflammasome inactivation and established a novel regulatory role of the Nrf2 pathway in ROSinduced NLRP3 priming.

Conclusions: We demonstrated Nrf2 negatively regulating NLRP3 inflammasome activity by inhibiting the priming step and suggested that Nrf2 could be a potential target for some uncontrolled inflammasome activation-associated diseases. Antioxid. Redox Signal. 26, 28-43.
\end{abstract}

Keywords: Nrf2, NLRP3 inflammasome, ROS, priming

\section{Introduction}

$\mathbf{T}$ HE NLRP3 Inflammasome is a multiprotein complex composed of the NLRP3 scaffold, ASC adaptor, and procaspase-1 (38). Recent studies indicate that the NLRP3 inflammasome can be activated when stimulated by both endogenous "danger signals" and compounds associated with pathogens (39). The assembly of the inflammasome leads to the maturation and secretion of some proinflammatory cytokines, such as IL-1 $\beta$ and IL-18 $(24,25)$. Moreover, the NLRP3 inflammasome has been regarded as a key component of innate immunity. However, excessive and chronic activation of the inflammasome results in many human diseases, such as fa- milial cold autoinflammatory syndrome (FCAS), cancer, type II diabetes (14), gout, atherosclerosis (22), and Alzheimer's disease (5). Thus, activation of the NLRP3 inflammasome should be effectively controlled (13).

Many studies have shown that activation of the NLRP3 inflammasome requires two steps. At first, pattern recognition or cytokine receptor-induced priming signal leads to the synthesis of NLRP3 and pro-IL- $1 \beta$ proteins. Then, a second signal promotes the NLRP3 inflammasome assemblymediated cleaved caspase- 1 and IL-1 $\beta$ secretion (3). The protein level of NLRP3 is critical for inflammasome assembly and regarded as a rate-limiting element for the inflammasome activation $(6,46)$. In resting macrophages,

Department of Physiology, State Key Laboratory of Natural Medicines, China Pharmaceutical University, Nanjing, China. 


\section{Innovation}

Nuclear factor erythroid 2-related factor 2 (Nrf2) activation-induced NQO1 expression contributed to the inhibition of NLR family, pyrin domain containing 3 (NLRP3) inflammasome activation in macrophages. In addition, tert-butylhydroquinone negatively regulated NLRP3 transcription by enhancing the antioxidant capacity in an Nrf2-antioxidant response element (ARE)dependent manner. As a reactive oxygen species scavenger, Nrf2-mediated NQO1 expression blocked NLRP3 priming, followed by attenuated activation of NLRP3. These results established a link between the Nrf2-ARE pathway and the NLRP3 inflammasome. These results also suggested that pharmacological activation of Nrf2 could be a potential therapeutic strategy for NLRP3associated diseases.

NLRP3 expression is relatively low and the assembly of the inflammasome complex is poorly induced. Many exogenous and endogenous factors can promote NLRP3 expression, including TLR agonists, proinflammatory cytokines, and reactive oxygen species (ROS) (37). It is interesting to note that almost all NLRP3 agonists can trigger ROS generation and that oxidative stress plays a vital role in NLRP3 inflammasome activation.

Redox signaling is a critical mechanism that mediates NLRP3 inflammasome activation. Changes in the redox microenvironment regulate the NLRP3 inflammasome activation resulting in cleaved caspase- 1 and mature IL- $1 \beta$ secretion (35). In addition, in vitro suppressing of ROS production by some scavengers or knockdown of NADPH oxidase subunits revealed that when ROS production was absent, NLRP3induced IL- $1 \beta$ generation was impaired, which indicated that ROS played crucial roles in inflammasome activation (10).

Notably, as a redox-sensitive transcription factor, Nrf2 regulates cellular antioxidant responses through modulating many phase II antioxidants, detoxifying enzymes, and some stress-responsive proteins $(12,20,42)$. Under basal conditions, Kelch ECH associating protein 1 (Keap1), a repressor protein, tethers Nrf2 to the actin cytoskeleton within the cytosol. The interaction between Nrf2 and Keap1 leads to continual ubiquitination and degradation of Nrf2. Under cellular stress conditions, Nrf2 is accumulated and translocated to the nucleus, binding to the antioxidant response element (ARE) to initiate the antioxidant response, which protects against a variety of reactive toxicants, including mitochondrial and NADPH oxidase-induced ROS $(28,23,45)$. The protective roles of Nrf2 have been reported in asthma (34), sepsis (44), and cigarette smoke-induced emphysema (43). Furthermore, sulforaphane has been demonstrated to ameliorate DSSinduced colitis (48), to which $\mathrm{Nrf}^{-1-}$ mice are more susceptible (19). Although the effects of Nrf2 on the regulation of many inflammatory diseases are emerging, the mechanisms of which remain largely unknown.

In this study, we show that Nrf2 activation induced NQO1 expression to scavenge ROS, which then inhibited NLRP3 expression and subsequent caspase- 1 cleavage. We identified Nrf2 as an endogenous regulator of the NLRP3 inflammasome and illustrated the fine regulation of inflammasome activation under physiological conditions.

\section{Results}

\section{Nrf2 inhibited NLRP3 inflammasome activation}

To investigate the possible roles of Nrf2 in NLRP3 inflammasome activation, lipopolysaccharide (LPS)-stimulated THP-Ms were pretreated with tert-butylhydroquinone (tBHQ) before challenging them with adenosine triphosphate (ATP). As shown in Figure 1A and Supplementary Figure S1A and B (Supplementary Data are available online at www.liebertpub. com/ars), tBHQ dose dependently increased the protein level and nuclear translocation of Nrf2. NLRP3-mediated caspase-1 activation (Fig. 1B and Supplementary Fig. S1C) and IL-1 $\beta$ maturation (Fig. 1C) were inhibited by tBHQ in a dosedependent manner. Consistent with this, tBHQ treatment significantly attenuated NLRP3 expressions at both the mRNA and protein levels (Fig. 1B-E).

To test whether the inhibitory effect of $\mathrm{BH}$ Q on the activation of NLRP3 inflammasome was mediated by Nrf2, siRNA targeting Nrf2 was used to suppress endogenous Nrf2 expression (Fig. 1F). Knockdown of Nrf2 in THP-Ms significantly increased LPS+ATP-induced NLRP3 inflammasome activation (Fig. 1H, J, and Supplementary Fig. S1E). On the contrary, the NLRP3 inflammasome was poorly activated when Nrf2 was overexpressed (Fig. 1I and Supplementary Fig. S1F). Combined with tBHQ stimulation, transfection in THP-Ms with an Nrf2 overexpression plasmid resulted in a sharp decrease of IL- $1 \beta$ secretion (Fig. 1I, K and Supplementary Fig. S1F), while transfection with siRNA or plasmid alone had no effect on inflammasome activation (Fig. $1 \mathrm{G}$ and Supplementary Fig. S1F). Together, these observations suggested that $\mathrm{tBHQ}$-induced Nrf2 activation negatively regulated the NLRP3 inflammasome activation.

\section{Nrf2 inhibited the priming step of NLRP3 inflammasome activation}

A sufficient level of NLRP3 protein is necessary to form and activate the NLRP3 inflammasome. Given that Nrf2 inhibited NLRP3 activation, we tested whether Nrf2 suppressed NLRP3 expression. THP-Ms were pretreated with $\mathrm{tBHQ}$, which was then followed by LPS stimulation. tBHQ treatment remarkably attenuated LPS-induced NLRP3 expression at both the protein (Fig. 2A and Supplementary Fig. S2A) and mRNA levels (Fig. 2B, C). In addition, stimulation of THP-Ms with tBHQ alone decreased NLRP3 expression (Fig. 2A). As shown in Figure 2D and G, Nrf2 knockdown greatly enhanced NLRP3 expression at both the mRNA and protein levels, while transfection with the Nrf2 plasmid had the opposite effect (Fig. 2E, $\mathrm{H})$. Taken together, these results indicated that Nrf2 activation inhibited LPS-induced NLRP3 production. It was previously reported that NF- $\kappa \mathrm{B}$ was inhibited by $\mathrm{Nrf} 2$ activation in certain inflammatory diseases (47). However, we were unable to observe any suppressive effects of tBHQ on NF- $\kappa \mathrm{B}$ nuclear translocation (Fig. 2F and Supplementary Fig. S2C). Traditional inflammasome studies adopt sequential stimulation methods, such as stimulating TLR ligands followed by a second stimulus several hours later to trigger NLRP3 inflammasome assembly. However, recent studies have indicated that cotreatment of LPS and ATP can induce the rapid activation of the NLRP3 inflammasome independent of the NLRP3 protein level (29). In this case, NLRP3 synthesis is not necessary for rapid caspase-1 activation. As shown in Figure 2I and Supplementary Figure S2D, the stimulation of THP-Ms with 
A

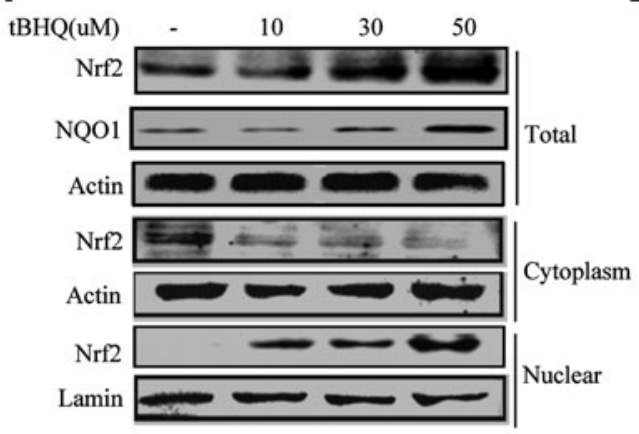

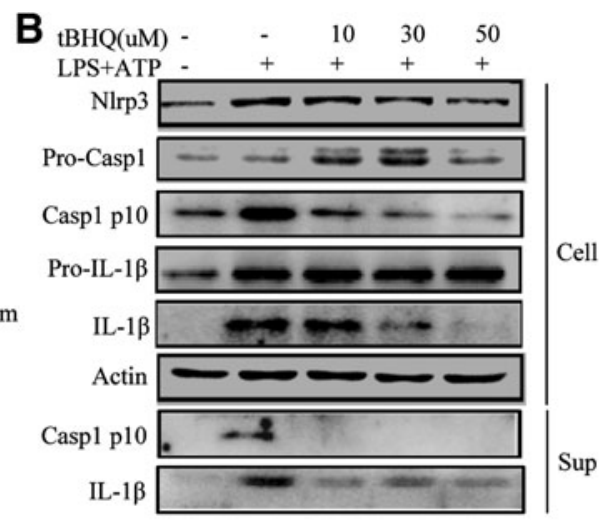

C

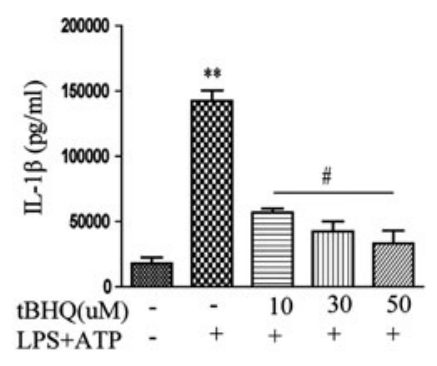

D

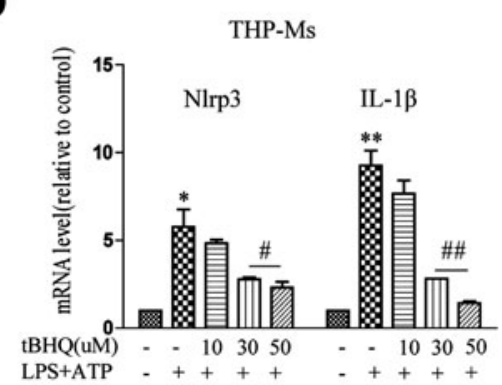

E

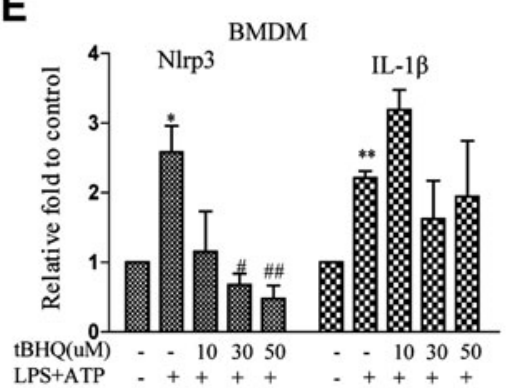

F $\quad$ trl siRNA

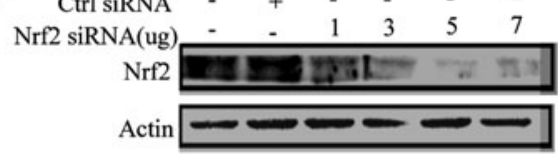

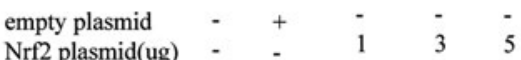
Nrf2 $-\infty-\infty$
Actin

H

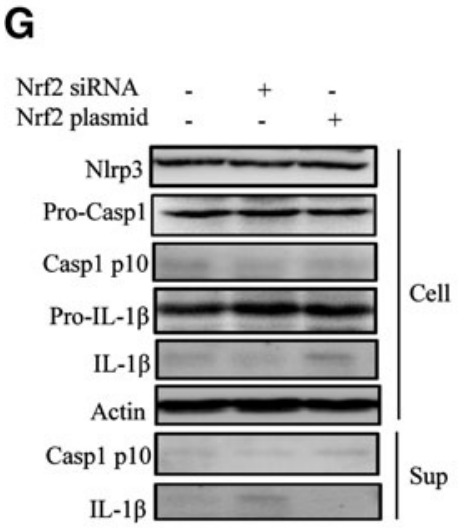

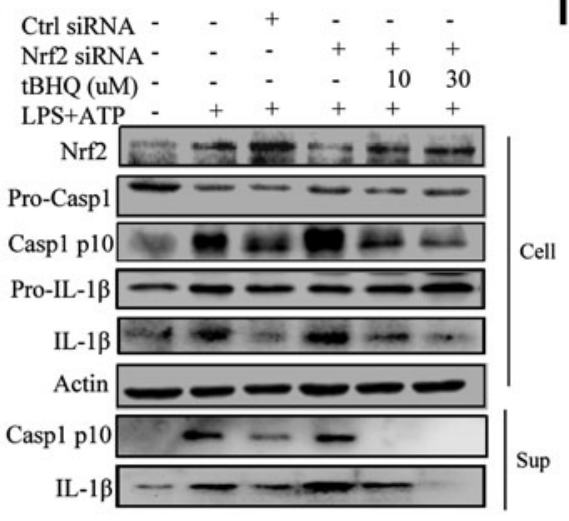

I

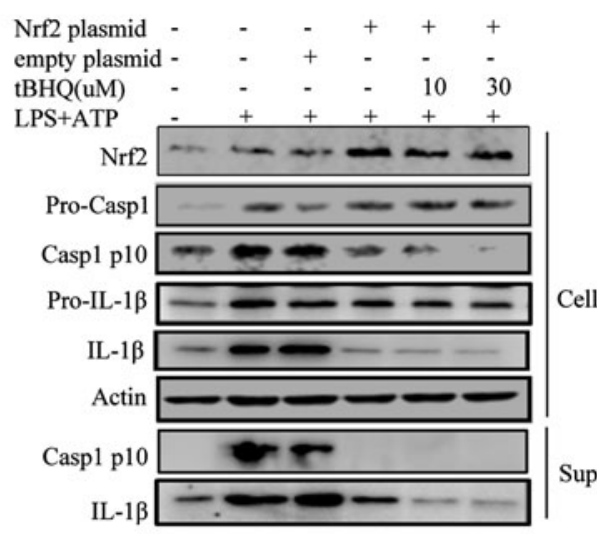

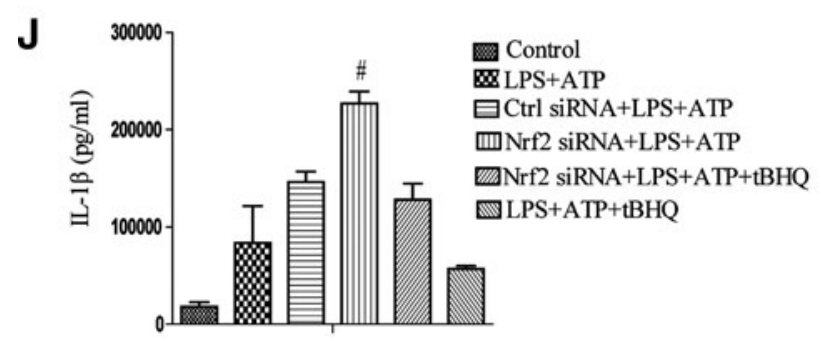

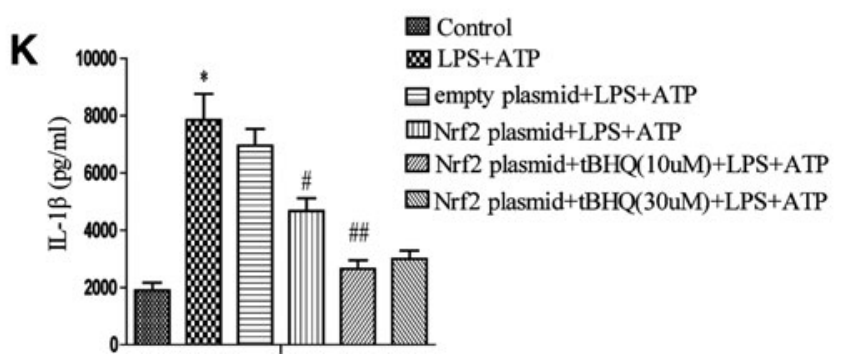

FIG. 1. tBHQ inhibits NLRP3 inflammasome activation. THP-Ms were treated with tBHQ for $12 \mathrm{~h}$, and the protein level of Nrf2 and its nuclear translocation were determined by Western blot (A). tBHQ-pretreated THP-Ms were stimulated with LPS for $4 \mathrm{~h}$ and ATP for $1 \mathrm{~h}$. The panel shows a Western blot of NLRP3, cleaved caspase-1 (p10), and cleaved IL-1 $\beta$ (p17) in the supernatants or cell lysates (B). IL-1 $\beta$ was measured by ELISA (C). The mRNA levels of NLRP3 and pro-IL-1 $\beta$ were detected by real-time RT-PCR (D, E). Western blot analysis of Nrf2 expression in THP-Ms transfected with different concentrations of Nrf2 siRNA or Nrf2 plasmid for $12 \mathrm{~h}(\mathbf{F})$. NLRP3, cleaved caspase-1, and IL-1 $\beta$ were detected by Western blot (G). Nrf2 siRNA-transfected THP-Ms were stimulated with LPS and ATP for the indicated periods. NLRP3, cleaved caspase-1, and IL-1 $\beta$ were detected by Western blot $\mathbf{( H )}$. IL-1 $\beta$ was measured by ELISA (J). Nrf2 plasmid-transfected THP-Ms were stimulated with LPS and ATP for the indicated periods. NLRP3, cleaved caspase-1, and IL- $1 \beta$ were detected by Western blot (I). IL-1 $\beta$ was detected by ELISA (K). The results are representative of three independent experiments and are expressed as the mean \pm SD. ${ }^{*} p<0.05$, $* * p<0.01$ compared with the control, ${ }^{*} p<0.05,{ }^{\# \#} p<0.01$ compared with LPS + ATP. ATP, adenosine triphosphate; LPS, lipopolysaccharide; NLRP3, NLR family, pyrin domain containing 3; Nrf2, nuclear factor erythroid 2-related factor 2; tBHQ, tert-butylhydroquinone; THP-Ms, THP-1 cell-derived macrophages. 

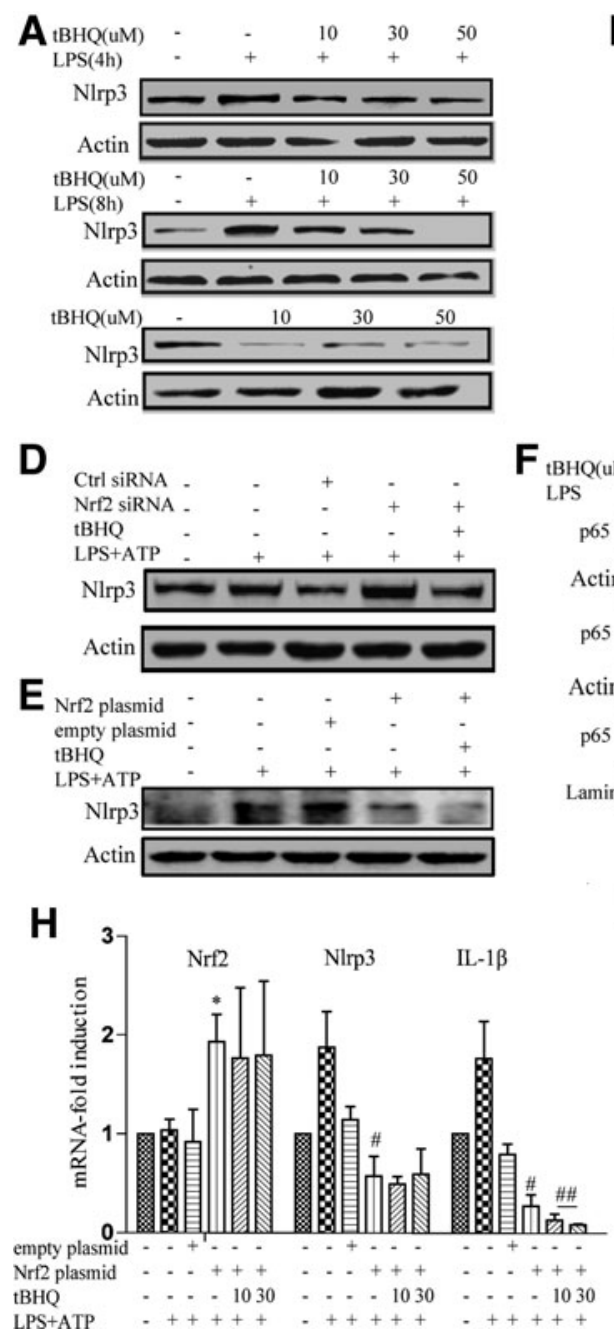
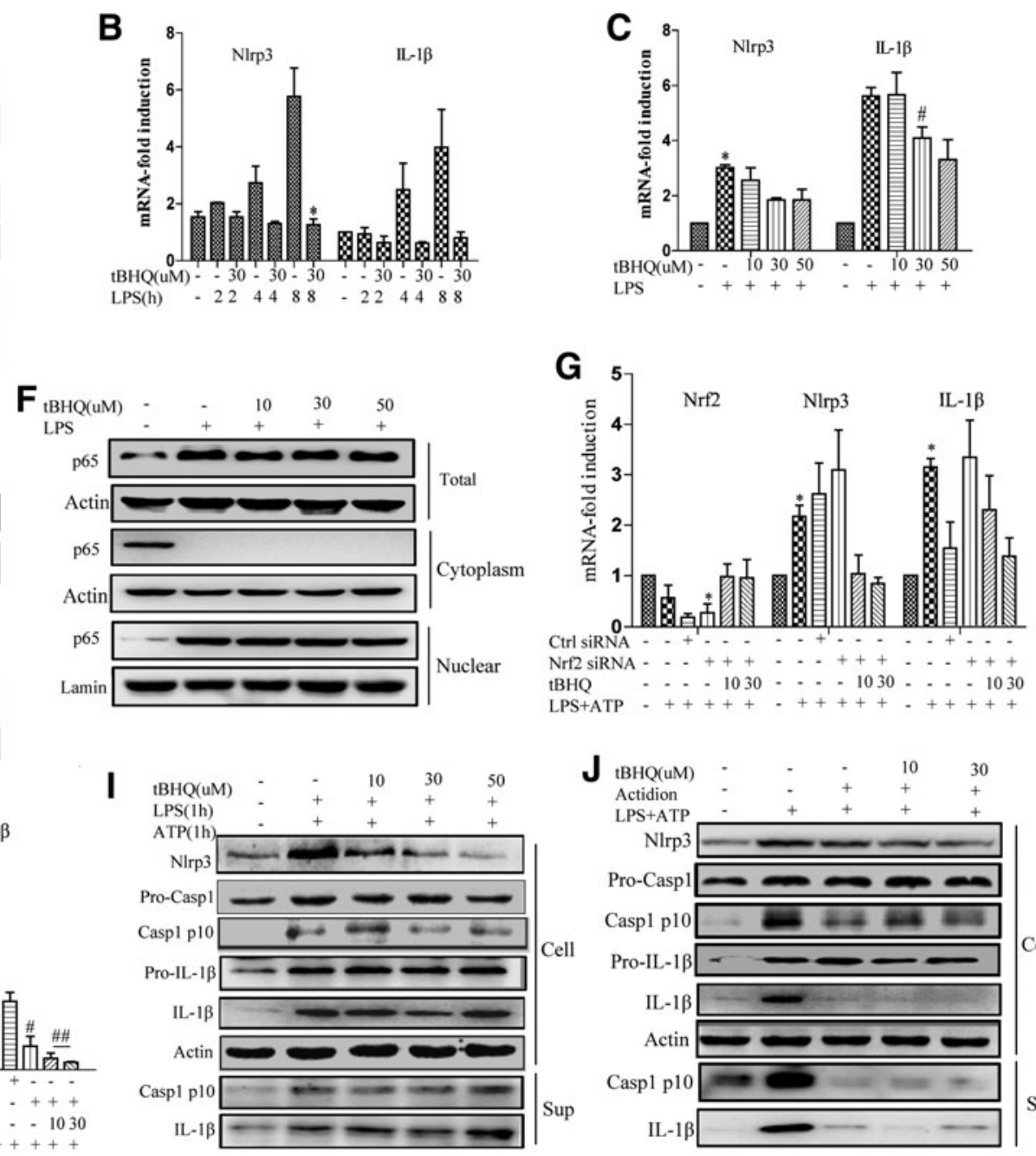
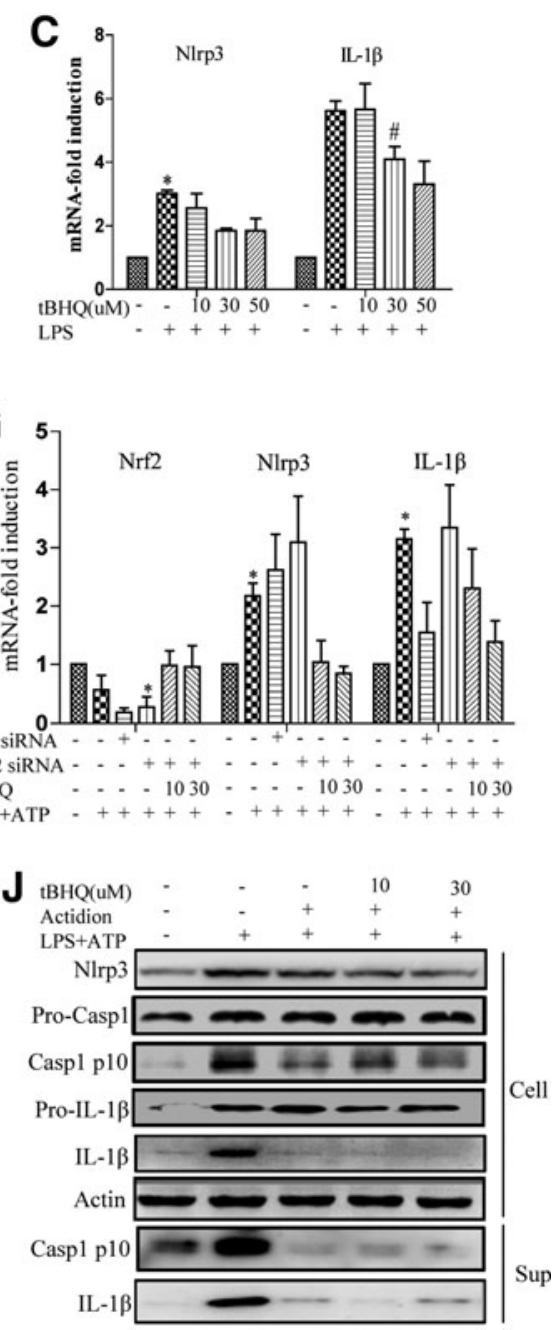

FIG. 2. Nrf2 inhibited the priming step of NLRP3 inflammasome activation. THP-Ms were pretreated with tBHQ for $12 \mathrm{~h}$ and then stimulated with or without LPS for the indicated periods. NLRP3 was detected by Western blot (A). The mRNA levels of NLRP3 and pro-IL-1 $\beta$ were measured by real-time RT-PCR (B, C). THP-Ms were transfected with Nrf2 siRNA or Nrf2 plasmid and then stimulated with LPS or ATP. The protein levels of NLRP3 were detected by Western blot (D, E). The mRNA levels of NLRP3 and pro-IL-1 $\beta$ were measured by real-time RT-PCR (G, H). tBHQ-pretreated THP-Ms were stimulated with LPS for $4 \mathrm{~h}$, and the protein level of NF- $\kappa \mathrm{B}$ and its nuclear translocation were determined by Western blot (F). tBHQ-pretreated THP-Ms were stimulated with LPS together with ATP for $1 \mathrm{~h}$. Cleaved caspase-1 (p10), IL-1 $\beta$, and NLRP3 were detected by Western blot (I). tBHQ-stimulated THP-Ms were pretreated with actidione for $1 \mathrm{~h}$ and then stimulated with LPS and ATP. Cleaved caspase-1 (p10), IL-1 $\beta$, and NLRP3 were detected by Western blot (J). The results are representative of three independent experiments and are expressed as the mean $\pm \mathrm{SD}$. $* p<0.05$, compared with the control, ${ }^{\#} p<0.05,{ }^{\# \#} p 0.01$ compared with LPS + ATP.

LPS and ATP for $1 \mathrm{~h}$ led to rapid NLRP3 inflammasome activation, while tBHQ treatment had no significant effect on NLRP3 inflammasome activation. Furthermore, when actidione was given before LPS stimulation to inhibit NLRP3 protein synthesis, the inhibitory effect of tBHQ on inflammasome activation was abolished (Fig. 2J and Supplementary Fig. S2E), indicating that Nrf2 inhibited NLRP3 inflammasome activation by suppressing NLRP3 transcription.

\section{tBHQ decreased the production of ROS induced by LPS and ATP}

Nrf2 plays a vital role in protection against oxidative stress. Previous reports identified that ROS were critically involved in NLRP3 inflammasome activation (35). Therefore, we mea- sured the production of ROS in THP-Ms under different stimulation conditions. tBHQ decreased total ROS in a timeand dose-dependent manner (Fig. 3A, B). ROS generation induced by LPS and ATP was also inhibited by tBHQ stimulation (Fig. 3C, D). Furthermore, Nrf2 siRNA caused higher ROS release (Fig. 3E), while a lower ROS level was observed in the context of Nrf2 overexpression (Fig. 3F). Similar results were also found in bone marrow-derived macrophages (BMDMs), where $\mathrm{Nrf}^{-/-}$BMDMs exhibited higher levels of ROS that could not be downregulated by tBHQ (Fig. 3G-I).

Superoxide is a component of mitochondrial ROS. Using flow cytometry, we examined the effect of tBHQ on the intracellular superoxide level. It was found that most of the intracellular superoxide elevation caused by LPS and ATP was decreased when Nrf2 was activated in advance (Fig. 4A). 

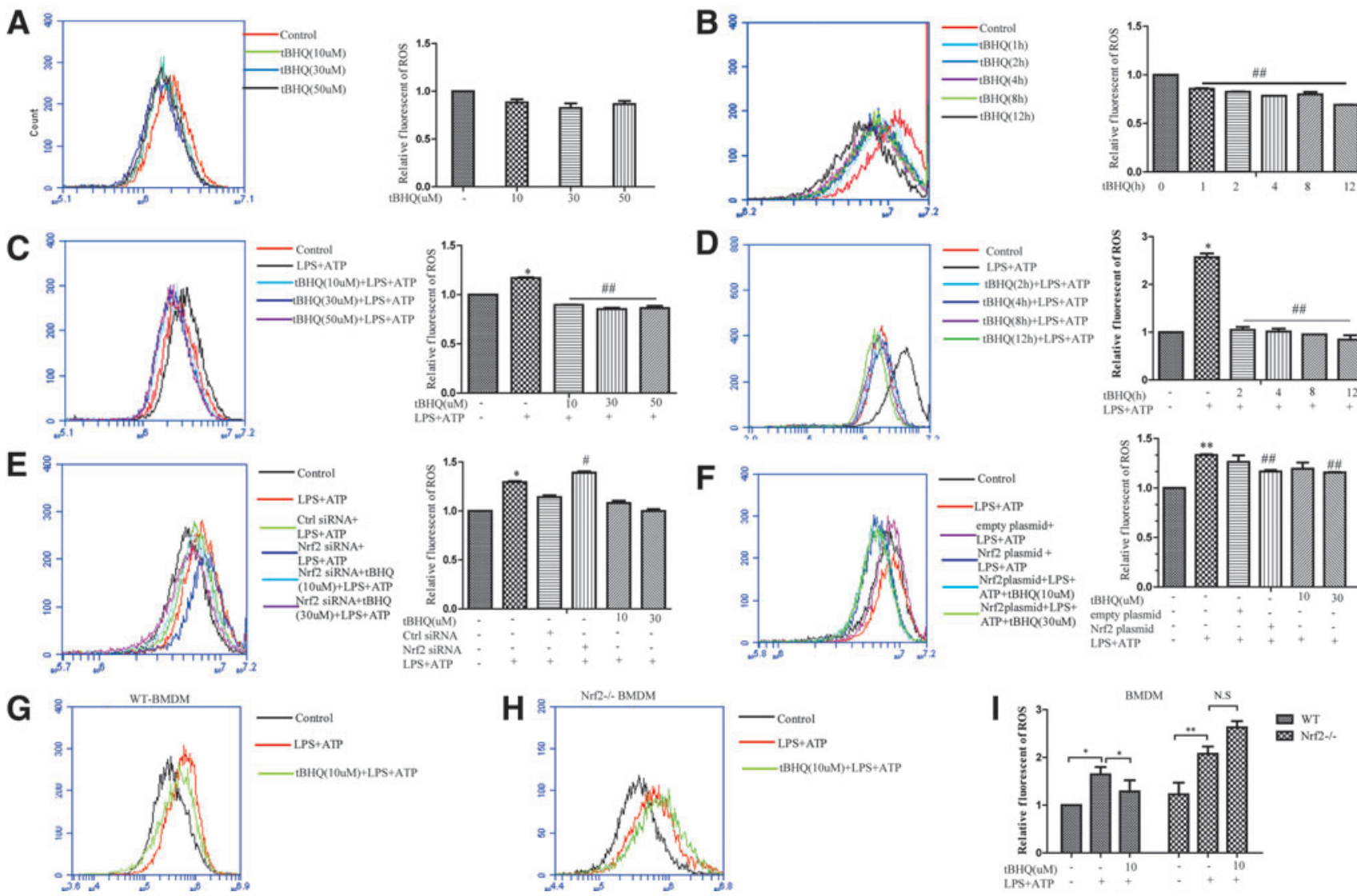

FIG. 3. tBHQ decreased the production of ROS induced by LPS and ATP. THP-Ms were stimulated by tBHQ with different doses for the indicated time, and the intracellular level of total ROS was measured by flow cytometry (A, B). Cells pretreated with different concentrations of tBHQ were stimulated by LPS and ATP; then, ROS were measured (C, D). Nrf2 siRNA and Nrf2 plasmid-transfected THP-Ms were stimulated by LPS and ATP at the indicated time and dose; then, the intracellular level of total ROS was measured by flow cytometry $(\mathbf{E}, \mathbf{F})$. WT BMDMs and $\mathrm{Nrf}^{-/}$BMDMs were stimulated by tBHQ, and the intracellular level of total ROS was measured by flow cytometry $(\mathbf{G}-\mathbf{I})$. The results are representative of three independent experiments and are expressed as the mean $\pm \mathrm{SD} .{ }^{*} p<0.05,{ }^{* *} p<0.01$ compared with the control, ${ }^{\#} p<0.05,{ }^{\# \#} p<0.01$ compared with LPS + ATP. N.S means no significant difference. BMDM, bone marrow-derived macrophage; ROS, reactive oxygen species. To see this illustration in color, the reader is referred to the web version of this article at www.liebertpub.com/ars

Moreover, superoxide was markedly enhanced in Nrf2deficient cells and inhibited by Nrf2 overexpression (Fig. 4B). The activity of superoxide dismutase (SOD) was also measured after different stimulation, which was found to be closely related to Nrf2 activation (Fig. 4C, D). Similar phenomena were observed in the levels of peroxide (Fig. 4E, F) and catalase (Fig. 4G, H). Taken together, these results strongly suggested that Nrf2 activation enhanced the activities of SOD and catalase to scavenge different sources of ROS.

\section{ROS promoted NLRP3 activation by affecting the priming step}

The above results suggested that tBHQ suppressed ROS production and NLRP3 inflammasome activation. To investigate the role of ROS in the activation of the NLRP3 inflammasome, $\mathrm{H}_{2} \mathrm{O}_{2}$ and rotenone were used to upregulate total intracellular ROS (Fig. 5A). $\mathrm{H}_{2} \mathrm{O}_{2}$ represented an exogenous way to promote ROS production, while rotenone, a respiratory chain inhibitor, increased mitochondrial ROS and enhanced total ROS. We found that ROS can both induce NLRP3 transcription and promote LPS-induced NLRP3 expression at the mRNA and protein levels (Fig. 5B, C). When NAC or rotenone was given to LPS-treated THP-Ms for $3 \mathrm{~h}$ followed by ATP stimulation, the production of both IL- $1 \beta$ (Fig. 5F) and cleaved caspase-1 (Fig. 5D) was decreased by NAC, whereas the activation of the NLRP3 inflammasome was increased by rotenone.

Based on the finding that ROS promoted the transcription of NLRP3 (Fig. 5B, C), we explored whether ROS primarily regulated the priming step. Rotenone was used as a substitute for either LPS or ATP to stimulate THP-Ms. As expected, both LPS and rotenone were able to prime the NLRP3 inflammasome, and when combined with ATP, they all induced cleaved caspase-1 activation. Hence, we speculated that ROS may play the same role as LPS in the activation of the NLRP3 inflammasome (Fig. 4E, G). Indeed, IL-1 $\beta$ secretion was not increased in response to LPS plus rotenone. That is, rotenone had little effect on the assembly of the NLRP3 inflammasome. Taken together, these results demonstrated that ROS promoted the priming step of NLRP3 inflammasome activation.

\section{The negative regulation of Nrf2 on the NLRP3 inflammasome was dependent on ROS}

Next, we study the underlying mechanism of the inhibitory effects of Nrf2 on NLRP3 inflammasome activation. Nrf2 

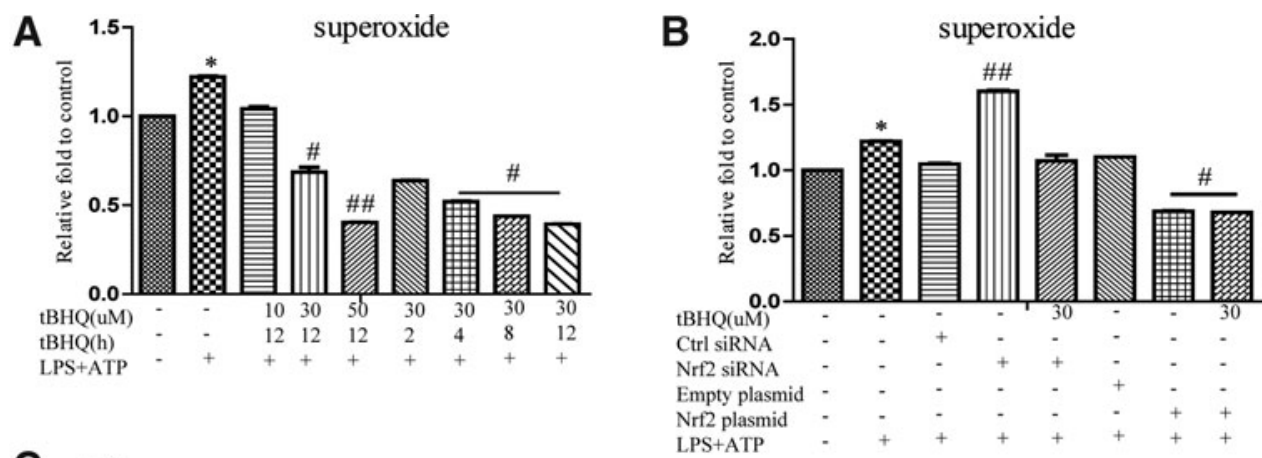

the activities of SOD and catalase to scavenge superoxide and peroxide. Cells pretreated with different concentrations of tBHQ were stimulated by LPS and ATP, and the intracellular levels of superoxide and peroxide were measured $(\mathbf{A}, \mathbf{E})$. Nrf2 siRNA or Nrf2 plasmidtransfected THP-Ms were stimulated by LPS and ATP at the indicated time and dose, and then, superoxide and peroxide were measured (B, F). THP-Ms were subjected to the above stimulation, and the activities of SOD $(\mathbf{C}, \mathbf{D})$ and catalase $(\mathbf{G}$, H) were detected. The results are representative of three independent experiments and are expressed as the mean $\pm \mathrm{SD}$. $* p<0.05$, compared with the control, ${ }^{\#} p<0.05,{ }^{\# \#} p<0.01$ compared with LPS + ATP. SOD, superoxide dismutase.
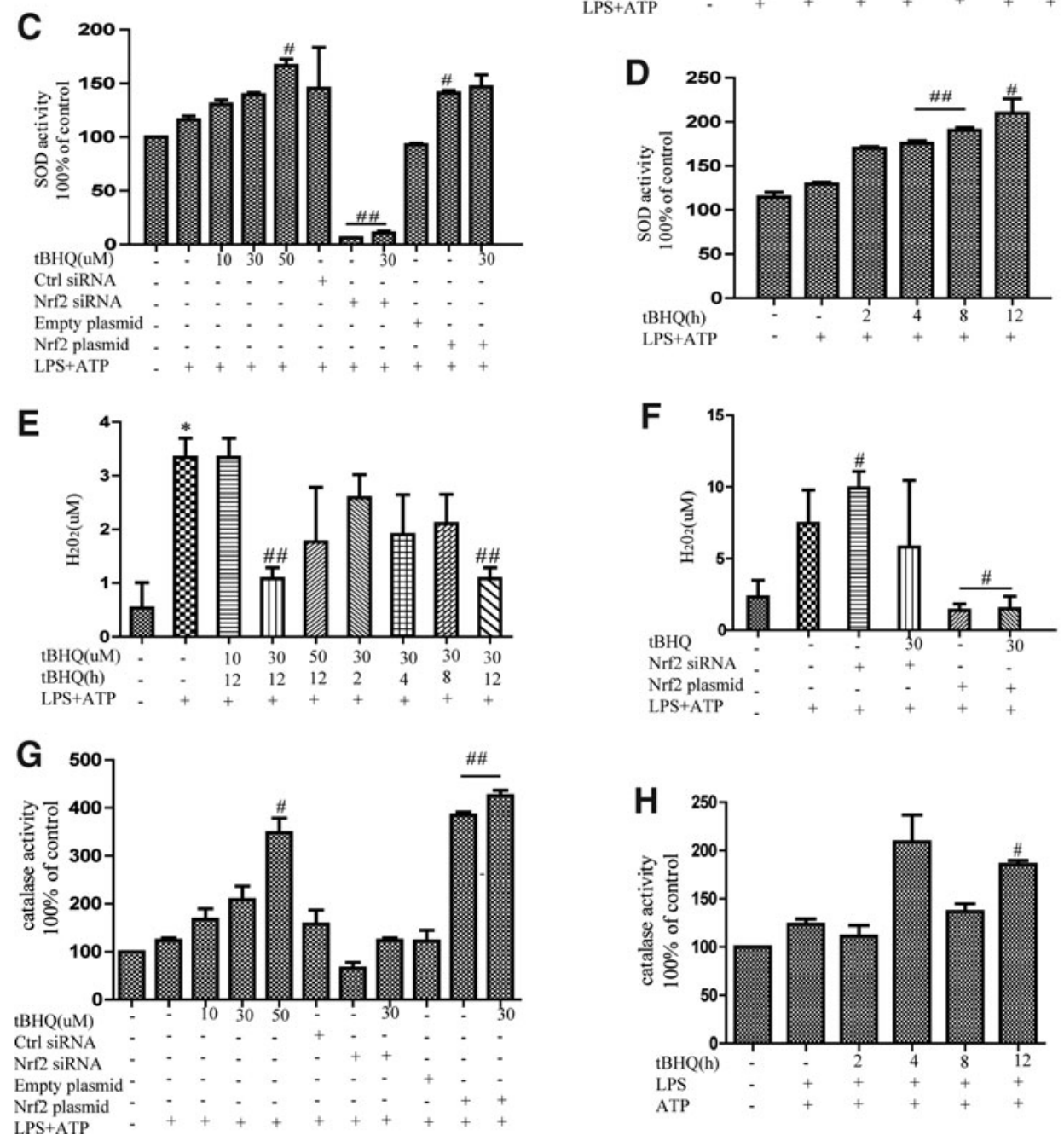

signaling suppressed ROS generation and caspase-1 activation. Hence, we tested the role of ROS in Nrf2-induced NLRP3 inflammasome inhibition, and it was found that the inhibitory effect of tBHQ on NLRP3 inflammasome activation was rescued by rotenone (Fig. 6A-C). Combined with ATP, $\mathrm{H}_{2} \mathrm{O}_{2}$ or rotenone (instead of LPS) could trigger NLRP3 inflammasome activation, which was inhibited by tBHQ pretreatment (Fig. 6D-I).

Moreover, transfection with Nrf2 siRNA increased intracellular ROS levels and promoted the transcription of NLRP3, cleaved casepase- 1 production and IL- $1 \beta$ secretion. This enhancement was significantly suppressed by NAC (Fig. 6J-L), suggesting that Nrf2-induced NLRP3 inflammasome inhibition was dependent on ROS. When transfected with the Nrf2 plasmid, neither rotenone nor NAC had any significant effect on NLRP3 inflammasome activation (Fig. 6M-O). We speculated that when Nrf2 was overexpressed, exogenous ROS were scavenged before activating the NLRP3 inflammasome. These data suggested that Nrf2 inhibited the activation of NLRP3 inflammasome by scavenging ROS.

The negative regulation of Nrf2 on NLRP3 inflammasome activation involved NQO1

As shown in Figure 1H, Nrf2 knockdown significantly increased LPS- and ATP-induced NLRP3 inflammasome activation. To further confirm this, the effect of tBHQ was tested in $\mathrm{Nrf}^{-/-}$BMDMs. The results showed that 
A

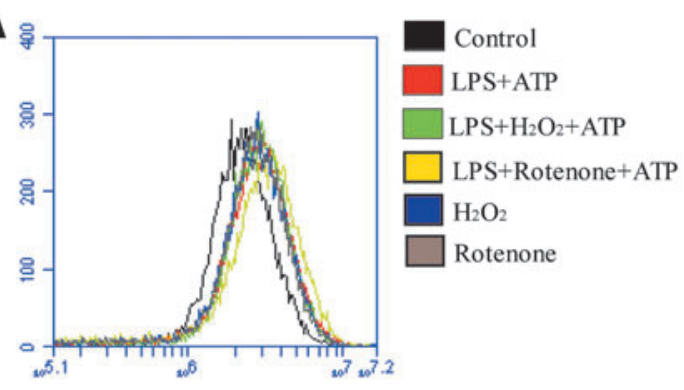

B

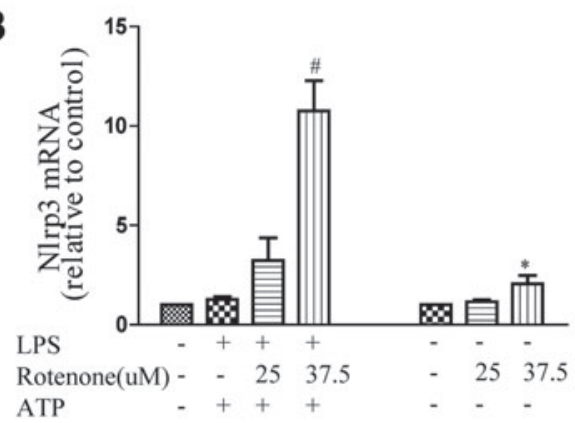

D NAC - $\quad-\quad+\quad-$

Rotenone - - - $+\quad+$

$\mathrm{H}_{2} \mathrm{O}_{2} \quad-\quad-\quad-\quad-+$

LPS+ATP - +++ -

Nlrp3 --

Pro-Caspl $-\square-\infty-\infty$

Caspl p10 $\div \ldots$

Pro-IL-1 $\beta$

IL-1 $\beta$

Actin

Casp1 p10

IL-1 $\beta$

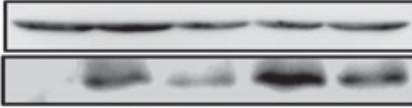

Cell

$\mathbf{F}$

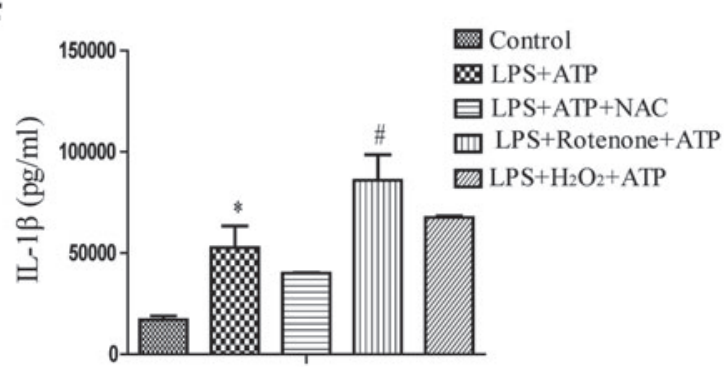

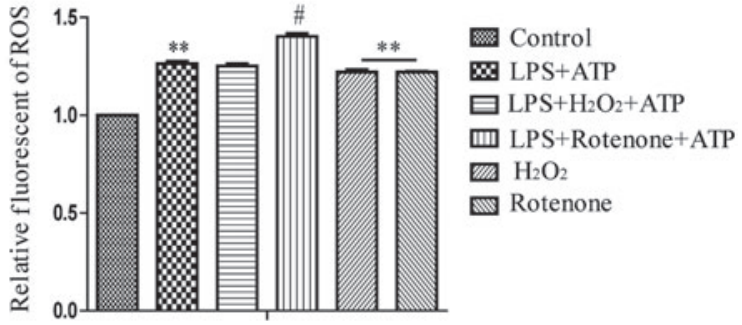

C

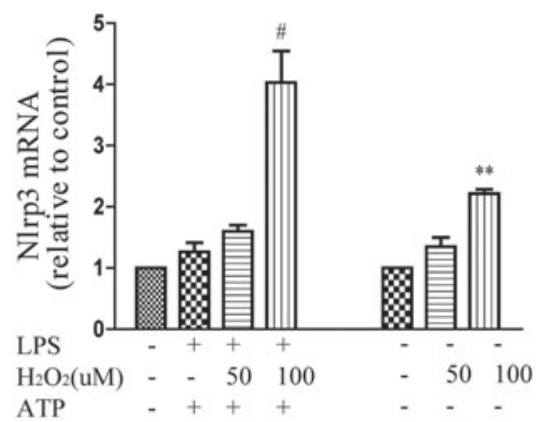

E

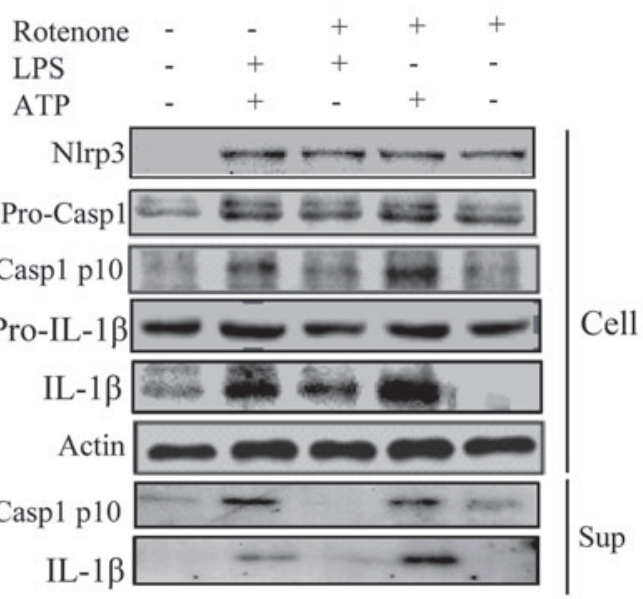

G

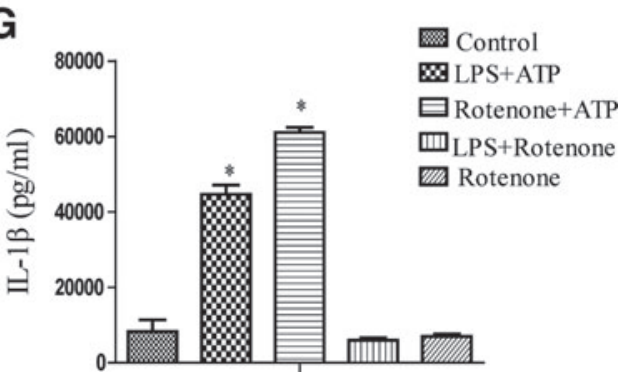

FIG. 5. ROS promoted NLRP3 activation by affecting the priming step. LPS-stimulated THP-Ms were exposed to NAC, $\mathrm{H}_{2} \mathrm{O}_{2}$, and rotenone and then treated with ATP. The intracellular level of ROS was measured by flow cytometry (A). The mRNA levels of NLRP3 and pro-IL- $1 \beta$ were measured by real-time RT-PCR $(\mathbf{B}, \mathbf{C})$. NLRP3, cleaved caspase-1, and IL-1 $\beta$ were detected by Western blot (D). IL-1 $\beta$ was detected by ELISA (F). Rotenone was substituted with LPS or ATP, and then, cleaved caspase-1, IL-1 $\beta$, and NLRP3 were detected by Western blot (E). IL-1 $\beta$ was detected by ELISA (G). The results are representative of three independent experiments and are expressed as the mean \pm SD. $* p<0.05$, $* * p<0.01$ compared with the control, ${ }^{\#} p<0.05$, compared with LPS + ATP. To see this illustration in color, the reader is referred to the web version of this article at www.liebertpub.com/ars 
A

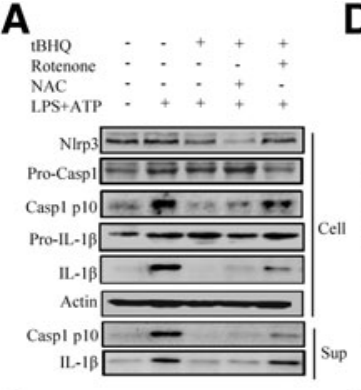

B

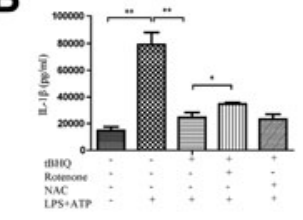

C

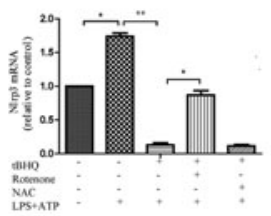

D

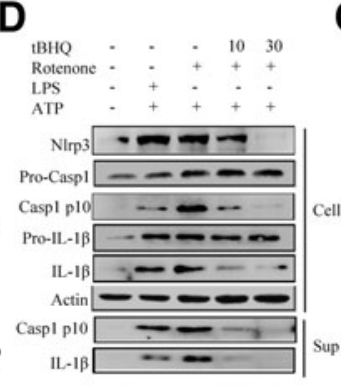

E

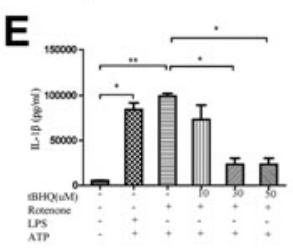

$\mathbf{F}$

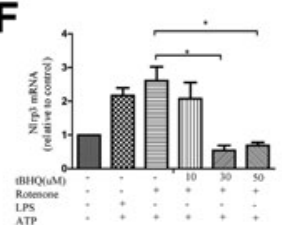

G

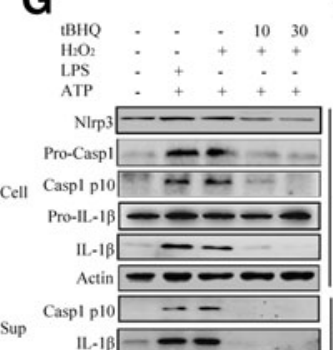

$\mathrm{H}$

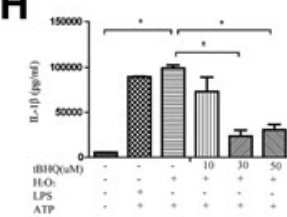

I

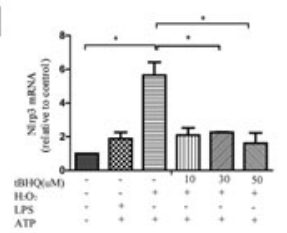

$\mathbf{J}$

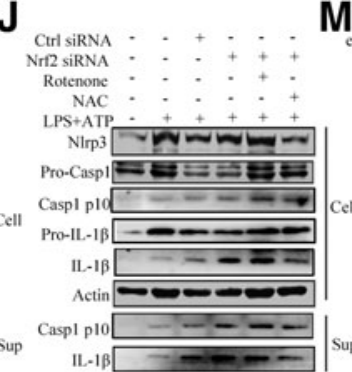

K

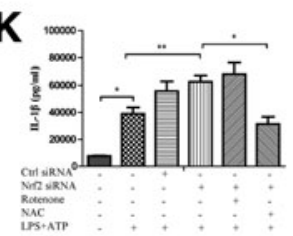

$\mathbf{L}$

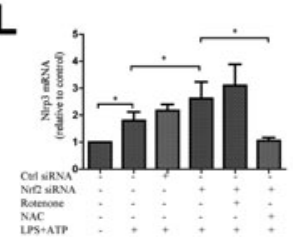

M

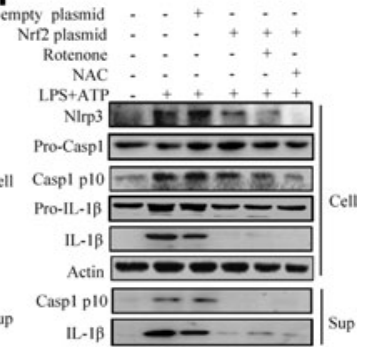

$\mathrm{N}$
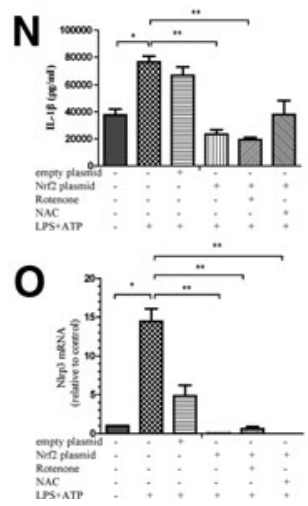

FIG. 6. The negative regulation of Nrf2 on the NLRP3 inflammasome was dependent on ROS. THP-Ms were pretreated with tBHQ for $12 \mathrm{~h}$ and then stimulated with LPS for $4 \mathrm{~h}$ and ATP for $1 \mathrm{~h}$. NAC or rotenone was added for the indicated time before ATP treatment. NLRP3, cleaved caspase-1, and IL-1 $\beta$ were detected by Western blot (A). IL-1 $\beta$ was detected by ELISA (B). The mRNA level of NLRP3 was detected by real-time RT-PCR (C). Western blot analysis of NLRP3, cleaved caspase-1, and IL-1 $\beta$ in tBHQ-pretreated cells stimulated with rotenone (D) or $\mathrm{H}_{2} \mathrm{O}_{2}$ (G) plus ATP. IL-1 $\beta$ was detected by ELISA (E, H). The mRNA level of NLRP3 was measured by real-time RT-PCR (F, I). Nrf2 siRNAtransfected THP-Ms were treated with the same stimulation. NLRP3, cleaved caspase-1, and IL- $1 \beta$ were detected by Western blot (J) IL-1 $\beta$ was detected by ELISA (K). The mRNA level of NLRP3 was measured by real-time RT-PCR (L). Nrf2 plasmid-transfected cells were shown (M-O). The results are representative of three independent experiments and are expressed as the mean \pm SD. ${ }^{*} p<0.05, * * p<0.01$.

the negative regulation of tBHQ on LPS+ATP-induced caspase-1 activation in BMDMs was abolished almost completely when Nrf2 was absent (Fig. 7A, B and Supplementary Fig. S3F, G). On the contrary, the Nrf2 overexpression plasmid significantly rescued the inhibitory function of tBHQ in $\mathrm{Nrf}^{-/-}$BMDMs (Fig. 7C, D, and Supplementary Fig. S3H). We found that treating BMDMs with different doses of tBHQ for $12 \mathrm{~h}$ increased the transcription of ARE genes, including GCLC, GCLM, GST, HO-1, and NQO1 (Fig. 7E). Notably, the transcription of NQO1 induced by tBHQ was significantly increased by 20 -fold. By contrast, in $\mathrm{Nrf}^{-/-}$BMDMs, the NQO1 mRNA level was not affected by tBHQ, while the mRNA levels of other ARE genes were increased by approximately fourfold when exposed to $50 \mu M$ tBHQ (Fig. 7F). In addition, in THP-Ms, the effect of tBHQ on the expression of NQO1, but not other ARE genes, was more profound than in Nrf2-silenced cells (Fig. 7G). A previous study reported that glutathione (GSH) may be the dominant factor in the cytoprotective actions of Nrf2 (11). However, we did not detect any remarkable effects of tBHQ on GSH in either THP-Ms or BMDMs (Supplementary Fig. S3I), suggesting that GSH may not be involved in the regulation of the NLRP3 inflammasome. Taken together, these data indicated that NQO1 was involved in the inhibitory effects of Nrf2 on NLRP3 inflammasome activation.

\section{$t B H Q$ attenuated alum-induced peritonitis in vivo}

To further test whether the in vitro biological effects of Nrf2 activation on NLRP3 inflammasome were also observable in vivo, an IL-1-dependent mouse peritonitis model was adopted. Mice received a gavage of tBHQ $(300 \mathrm{mg} / \mathrm{kg}$ and $600 \mathrm{mg} / \mathrm{kg}$ ) for 1 week followed by an intraperitoneal (i.p.) injection of alum. After that, we collected peritoneal exudate cells (PECs), and inflammatory cells recruited by alum were analyzed by flow cytometry. As shown in Figure 8A-D, alum-induced recruitment of $\mathrm{Ly} 6 \mathrm{G}^{+}$neutrophils and $\mathrm{Ly}^{+} \mathrm{C}^{+}$monocytes was significantly blocked by different doses of tBHQ. In addition, less caspase-1 cleavage and mature IL- $1 \beta$ secretion were observed in the lavage fluid from mice that received a gavage of tBHQ (Fig. 8E, F). Notably, we observed that alum-recruited PECs had a fusiform shape, rather than a circular shape, as observed in normal and tBHQ pretreatment groups (Fig. 8G).

However, $\mathrm{Nrf}^{-/-}$mice showed a remarkable increased recruitment of inflammatory cells, including $\mathrm{Ly}-6 \mathrm{G}^{+}$neutrophils and Ly-6C ${ }^{+}$inflammatory monocytes, after intraperitoneal (i.p.) injection of alum compared with wild-type mice (Fig. 9A-D), supporting a critical anti-inflammatory role of Nrf2 in alum-induced peritonitis. In addition, higher NLRP3 and cleaved caspase- 1 expression, as well as IL-1 $\beta$ secretion, were also observed in the lavage fluid from $\mathrm{Nrf} 2^{-l-}$ 

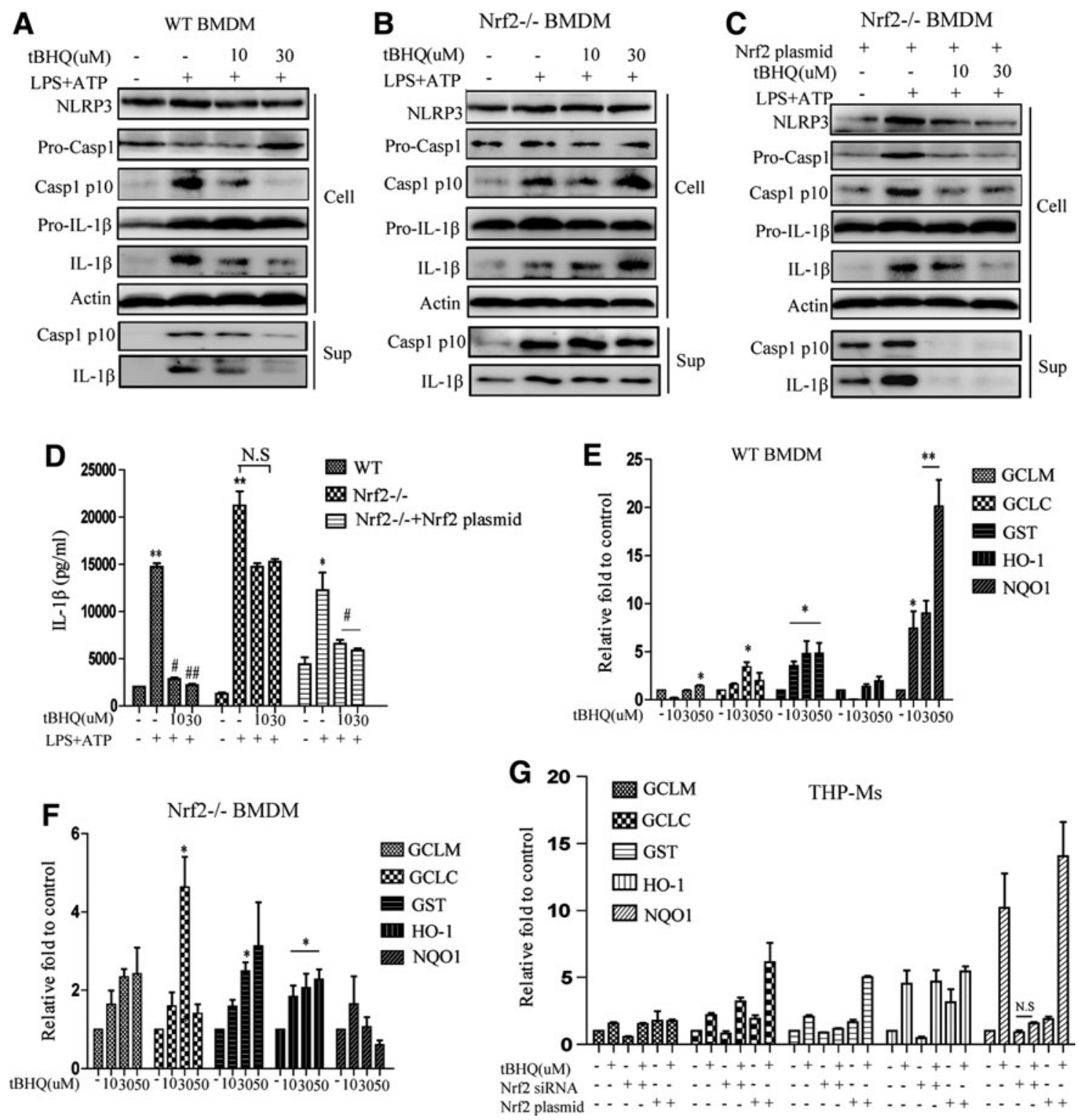

FIG. 7. The negative regulation of Nrf2 on NLRP3 inflammasome activation involved NQO1. tBHQ-pretreated WT BMDMs, Nrf2 ${ }^{-1-}$ BMDMs, and Nrf2 plasmid-transfected Nrf2 ${ }^{-l-}$ BMDMs were stimulated with LPS for $4 \mathrm{~h}$ and ATP for $1 \mathrm{~h}$, and then, NLRP3, cleaved caspase-1, and IL-1 $\beta$ were detected by Western blot (A-C). IL-1 $\beta$ was detected by ELISA (D). WT BMDMs and Nrf2 ${ }^{-1-}$ BMDMs were treated with tBHQ for $12 \mathrm{~h}$, and the mRNA levels of GCLM, GCLC, GST, HO-1, and NQO1 were measured by real-time RT-PCR $(\mathbf{E}, \mathbf{F})$. The expressions of the above mRNA were detected in THP-Ms treated with different stimulations $(\mathbf{G})$. The results are representative of three independent experiments and are expressed as the mean \pm SD. ${ }^{*} p<0.05, * * p<0.01,{ }^{*} p<0.05,{ }^{* \#} p<0.01$ compared with LPS + ATP, N.S means no significant difference.

mice (Fig. 9E, F). Nrf2 deficiency may abolish its protective effect against alum-induced peritonitis, as immune cells had a more mature phenotype and a fusiform shape (Fig. 9G). Overall, these findings further confirmed that Nrf2 activation inhibited NLRP3 inflammasome activity in vivo.

\section{Discussion}

Recently, Nrf2 and the NLRP3 inflammasome have been identified as regulators of many inflammatory diseases. They have also become useful targets of anti-inflammatory interventions for the treatment of liver injury (32), lupus nephritis (18), and colitis (49). More recently, Pei-Yi Tsai demon- strated that epigallocatechin-3-gallate had prophylactic effects on lupus nephritis by increasing the Nrf2 antioxidant signaling pathway and inhibiting renal NLRP3 inflammasome activation (11), which could also be applied to the treatment of glomerulonephritis (50). In addition, schisandrin B was reported to induce Nrf2 expression and attenuate ROSdependent peritonitis (26). Although studies have revealed that enhancing Nrf2 and suppressing NLRP3 can be used to treat inflammation, it is still unknown whether there is a crosslink between the Nrf2-ARE pathway and NLRP3 inflammasome and whether Nrf2 inducers could be adopted to protect against NLRP3-associated diseases. Further in-depth biochemical analysis about the interaction between Nrf2 and 
A
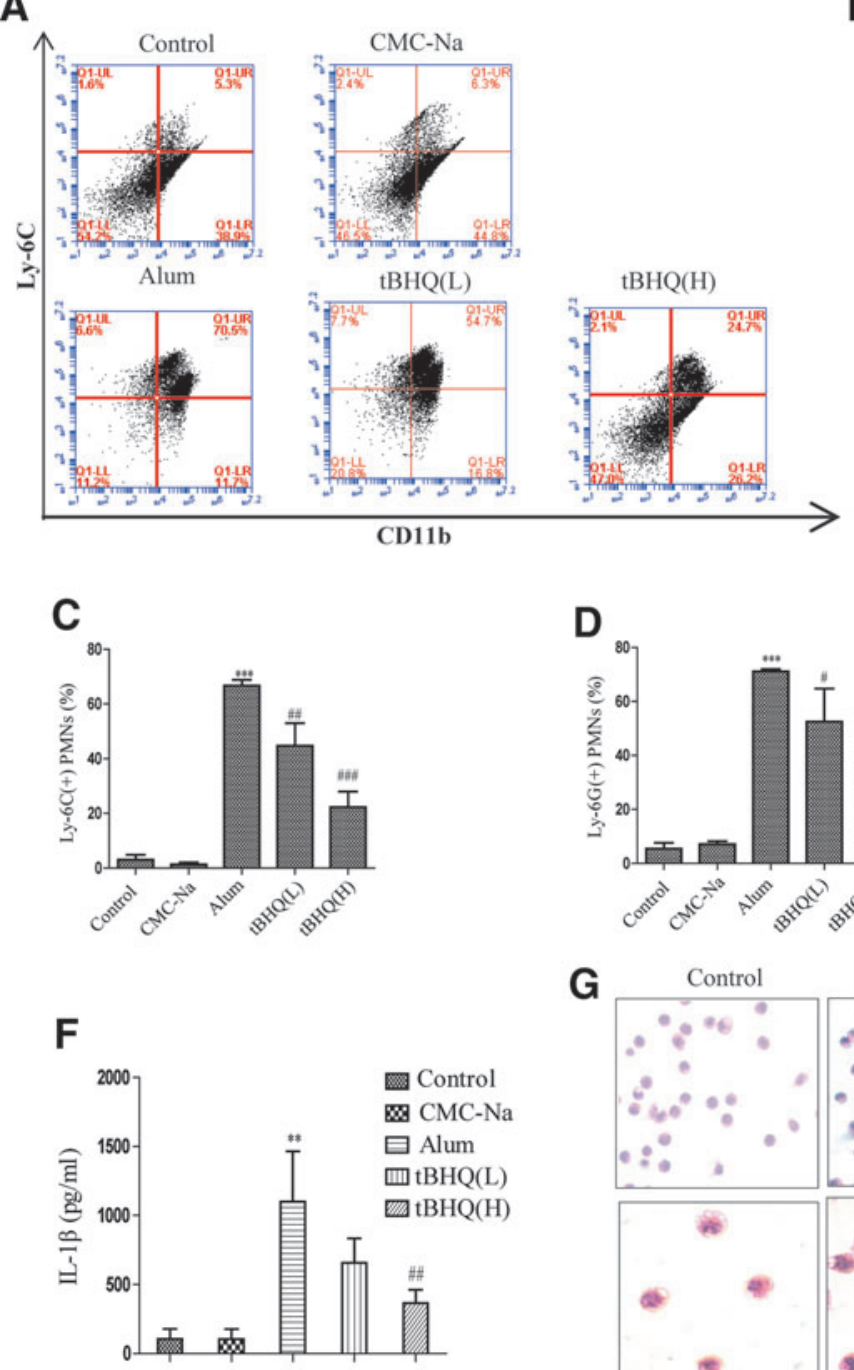

G
B
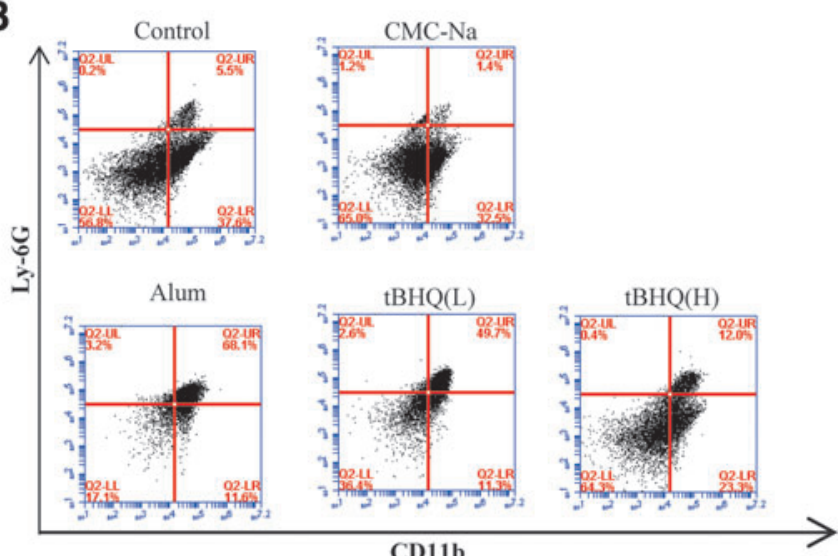

CD11b

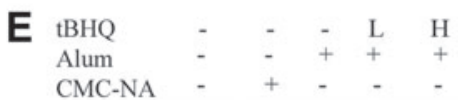

Nlrp3 $\rightarrow-20$
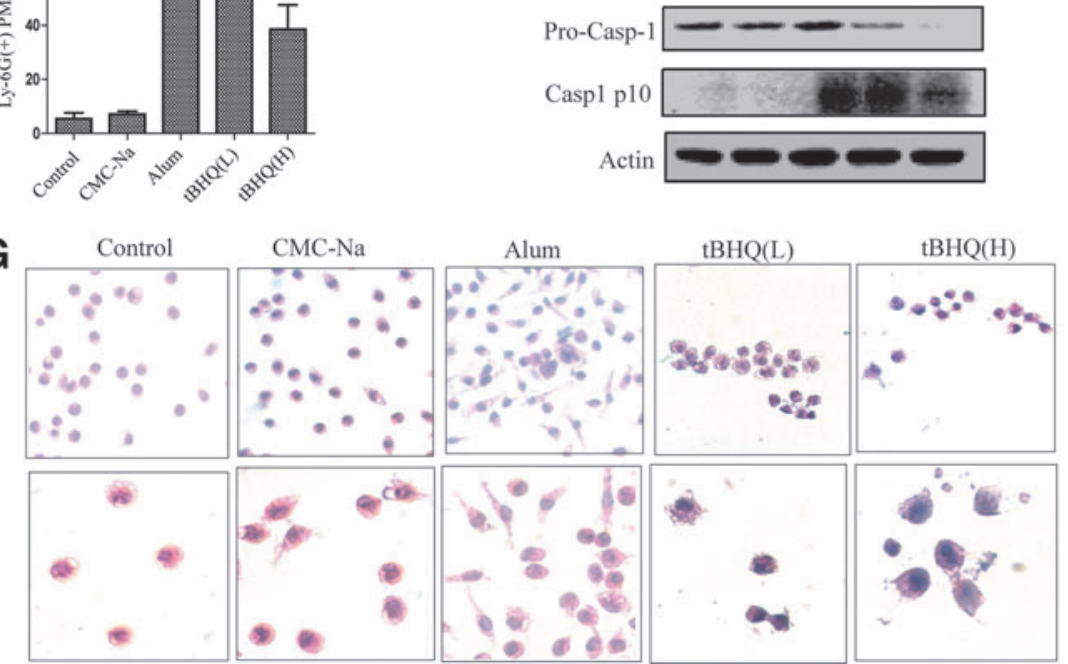

FIG. 8. tBHQ attenuated alum-induced peritonitis in vivo. Different groups of mice $(n=8)$ were intraperitoneally injected with alum ( $700 \mu \mathrm{g} / \mathrm{per}$ mouse) diluted in sterile PBS. Peritoneal lavage was performed for $12 \mathrm{~h}$ after injection. The ratio of $\mathrm{CD} 11 \mathrm{~b}^{+} \mathrm{Ly}-6 \mathrm{C}^{+}$inflammatory monocytes $(\mathbf{A}, \mathbf{C})$ and $\mathrm{CD} 11 \mathrm{~b}^{+} \mathrm{Ly}-6 \mathrm{G}^{+}$neutrophils $(\mathbf{B}, \mathbf{D})$ was evaluated by FACS analysis. PECs were seeded in 12-well plates and HE stained (G). The protein levels of NLRP3 and cleaved caspase-1 were detected by Western blot (E). IL-1 $\beta$ was detected by ELISA (F). The results are representative of at least three independent experiments and are expressed as the mean \pm SD. $* * p<0.01, * * * p<0.001$ compared with the control, ${ }^{\#} p<0.05,{ }^{\# \#} p<0.001$ compared with alum. PEC, peritoneal exudate cell. To see this illustration in color, the reader is referred to the web version of this article at www.liebertpub.com/ars

the NLRP3 inflammasome should provide mechanistic insights. In this study, we found that Nrf2 activation inhibited NLRP3 inflammasome activation by decreasing ROSinduced NLRP3 transcription.

Our in vivo study showed that $\mathrm{Nrf} 2^{-/-}$mice are more susceptible to peritonitis than wild-type mice, which does not agree with a recent study by Zhao et al. indicating that $\mathrm{Nrf} 2^{-/-}$mice have significantly less recruitment of immune cells and are less susceptible to peritonitis (51). Aluminduced peritonitis is regarded as a typical NLRP3-IL-1 $\beta$ axis-dependent inflammation animal model, and notably, the dose of alum and its stimulation time influence immune cell recruitment in the peritoneal cavity. The omentum is a major site of entry for leukocytes during peritoneal inflammation (8), and a sufficient dose of alum is required to instigate leukocyte recruitment. Generally, a dose of $700 \mu \mathrm{g}$ per mouse has been adopted in most experiments $(9,16)$. In addition, monocyte-derived macrophages are known to migrate from the peritoneum in the resolution phase of acute inflammation (4), and the longer time course would increase the migration of immune cells. In our study, we used an alum dose of $700 \mu \mathrm{g}$ per mouse for $12 \mathrm{~h}$ to induce acute peritonitis, which is different from Zhao's methodology $(400 \mu \mathrm{g}$ of alum per mouse for $16 \mathrm{~h}$ ). We speculate that the dose of alum used and the duration of the exposure may explain the contradiction between our study and Zhao's.

We hypothesized that Nrf2 preactivation leads to the inhibition of NLRP3 inflammasome activation via a ROSdependent mechanism. Indeed, tBHQ decreased the production of superoxide and peroxide, while the activities of SOD and catalase were significantly enhanced. Larry et al. demonstrated that Nrf2 played a critical role in controlling 
A

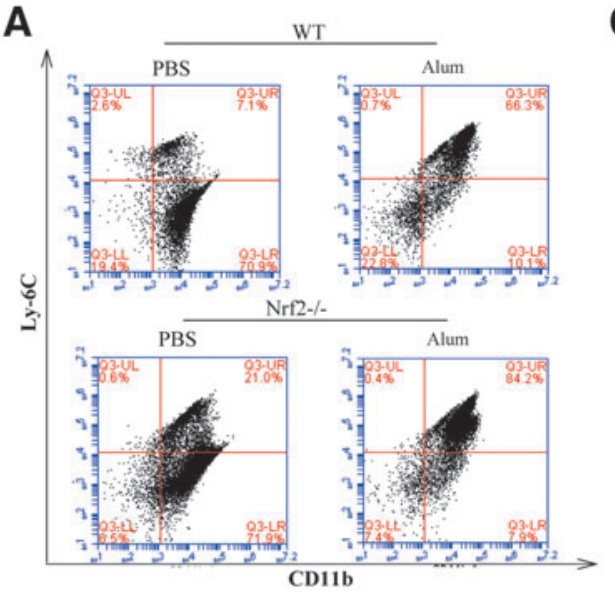

B

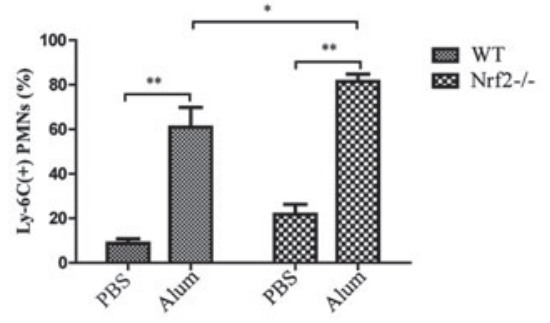

E

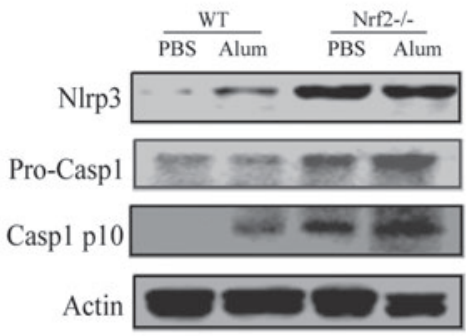

G

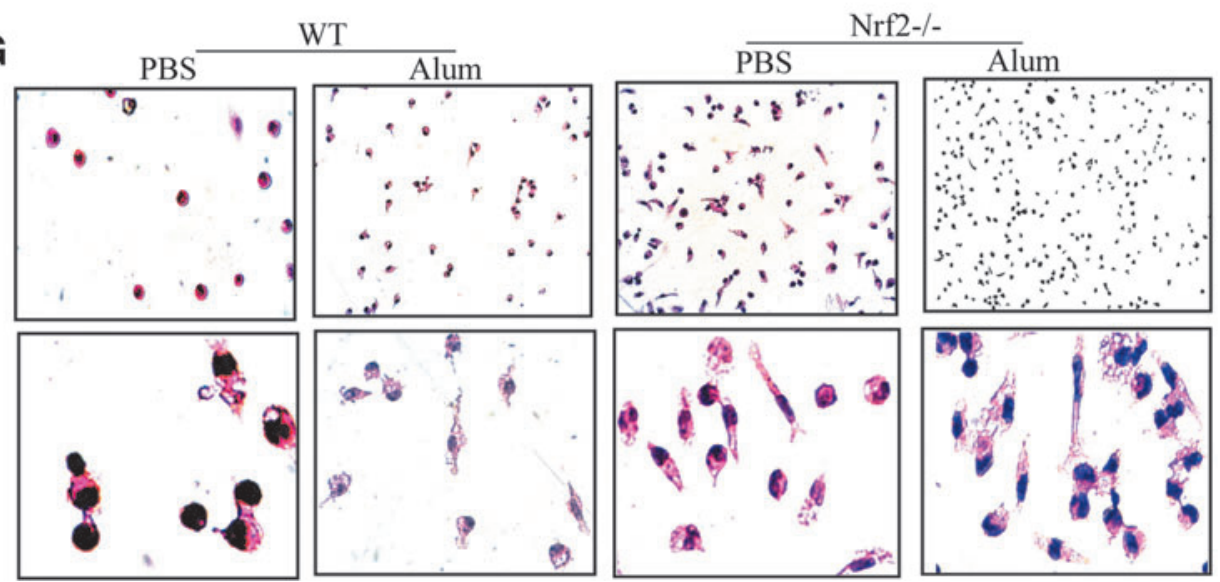

C
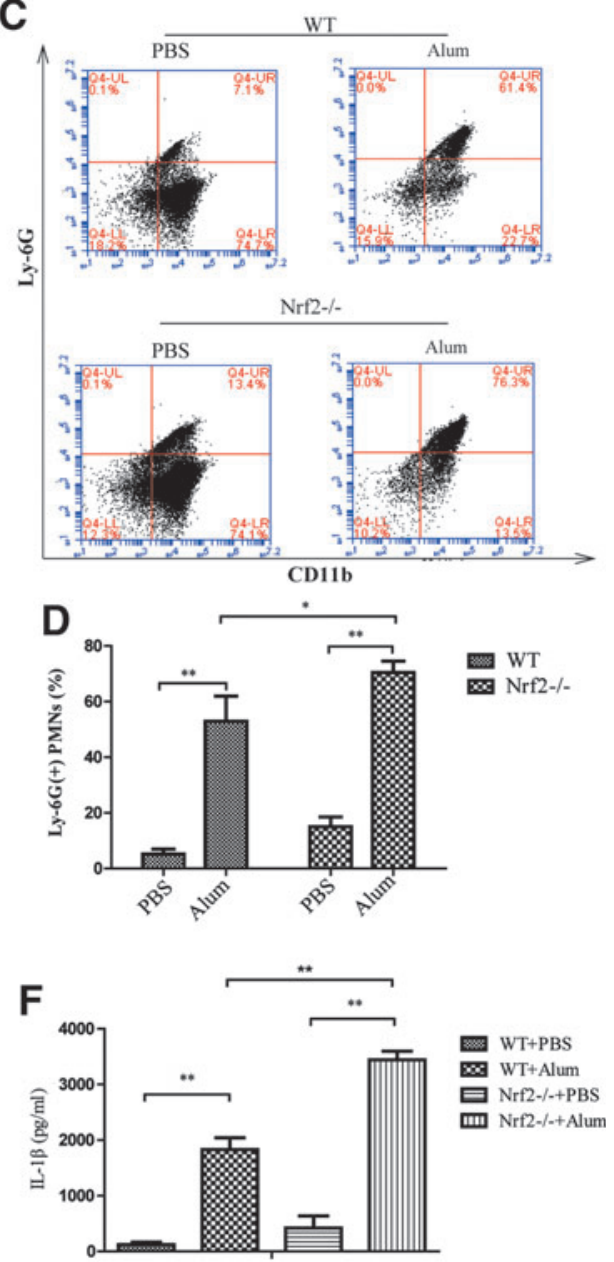

Nrf2-/-
FIG. 9. Enhanced aluminduced peritonitis in $\mathrm{Nrf2}^{-/-}$ mice. WT or Nrf $2^{-1-}$ mice $(n=8)$ were intraperitoneally injected with alum $(700 \mu \mathrm{g} /$ per mouse) diluted in sterile PBS. Peritoneal lavage was performed $12 \mathrm{~h}$ after injection. The ratio of $\mathrm{CD} 11 \mathrm{~b}^{+} \mathrm{Ly}-$ $6 \mathrm{C}^{+}$inflammatory monocytes $(\mathrm{A}, \mathrm{B})$ and $\mathrm{CD} 11 \mathrm{~b}^{+} \mathrm{Ly}-6 \mathrm{G}^{+}$ neutrophils $(\mathbf{C}, \mathbf{D})$ was evaluated by FACS analysis. Cytospin preparation followed by HE staining was performed (G). The protein levels of NLRP3 and cleaved caspase-1 were detected by Western blot (E). IL-1 $\beta$ was measured by ELISA (F). The results are representative of at least three independent experiments and are expressed as the mean $\pm \mathrm{SD} . * p<0.05$, $* * p<0.01$. To see this illustration in color, the reader is referred to the web version of this article at www.liebertpub .com/ars basal GSH levels by modulating the rate of GSH synthesis in mouse embryonic fibroblasts. Moreover, GSH was entirely responsible for the protection against a soft and hard electrophile, an organic hydroperoxide, and a redox-cycling agent, indicating that increasing the abundance of GSH is more important than the induction of enzymes, such as heme oxygenase-1 and $\mathrm{NAD}(\mathrm{P}) \mathrm{H}$ :quinone oxidoreductase-1, in scavenging ROS (11). In the present study, we measured the mRNA levels of Nrf2-targeted ARE genes, including GCLC, GCLM, GST, HO-1, and NQO1, and found that the effect of
tBHQ on the mRNA level of NQO1, but not other ARE genes, was more profound than that in Nrf2-deficient cells. Notably, the expression of NQO1 was closely related to Nrf2mediated inhibition of the NLRP3 inflammasome, which indicated that Nrf2-induced NQO1 expression played a critical role in scavenging ROS. In addition, NQO1 was identified as a highly inducible cytoprotective gene that regulated ROS generation (21). tBHQ could ameliorate arsenic-induced cytotoxicity and apoptosis by inducing Nrf2dependent antioxidant responses in which NQO1 was 
FIG. 10. Proposed model illustrating how Nrf2 negatively regulated NLRP3 inflammasome activation. ROS induced by LPS promoted NLRP3 transcription. Then, ATP stimulation activated the NLRP3 inflammasome, which led to mature IL- $1 \beta$ secretion. tBHQ induced the nuclear translocation of Nrf2, NQO1 expression, and scavenged intracellular ROS, which resulted in the decrease of NLRP3 transcription and subsequent inflammasome activation. To see this illustration in color, the reader is referred to the web version of this article at www.liebertpub.com/ars

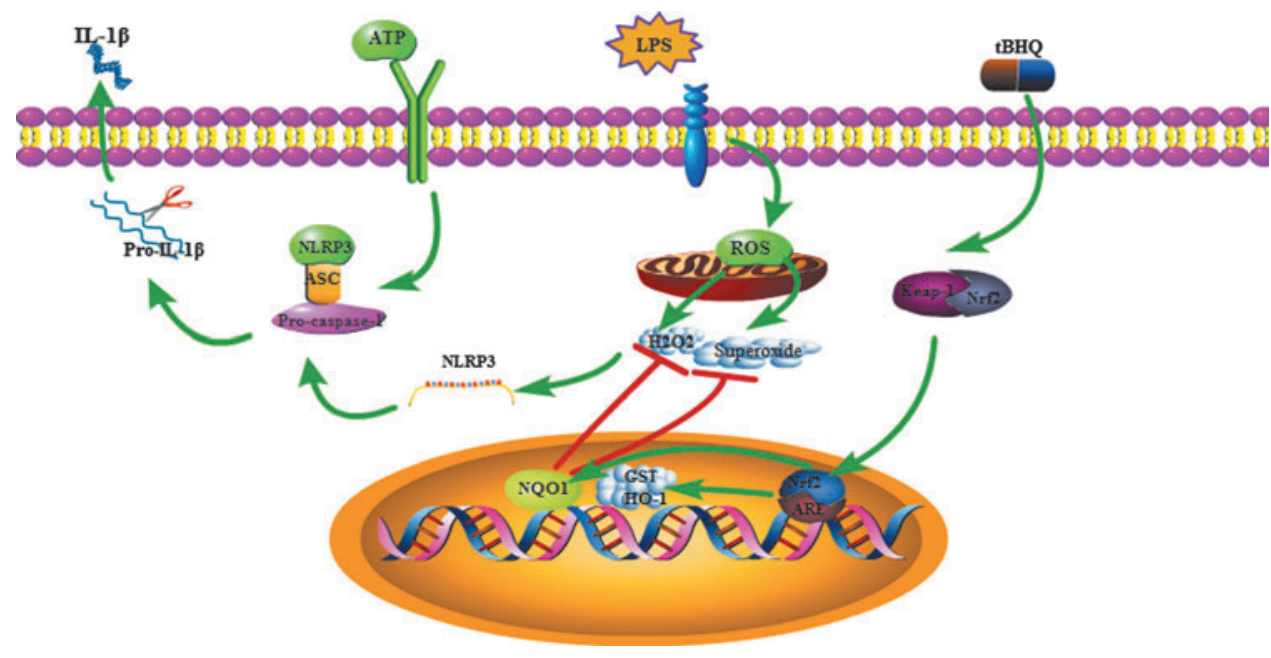

involved (7). In addition, the NQO1 inhibitor diminutol (Dim) abolished the suppressive function of Nrf2 on hepatotoxicity (33). Therefore, NQO1 may account for the inhibitory effects of Nrf2 on the regulation of NLRP3 inflammasome activation.

Oxidative stress is considered as a common feature of inflammatory responses, and ROS act as an upstream signal mediating the NLRP3 inflammasome activation $(36,54)$. In this study, our results showed that ROS exerted their effects by promoting the transcription of NLRP3. NF- $\kappa \mathrm{B}$ was reported to be an ROS-sensitive transcription factor that mediated NLRP3 inflammasome activation (27), but we did not find any change in the NF- $\kappa \mathrm{B}$ protein level or its nuclear translocation after exposure to tBHQ. Bauernfeind et al. (2) demonstrated that the priming step of NLRP3 inflammasome strictly requires ROS production, which is not necessary for inflammasome activation (1). Recently, a novel mechanism for ROS regulation of the post-translational activation of NLRP3 by deubiquitination has been put forward (17). In addition, ROS induced oxidized mitochondrial DNA release into the cytosol (40), where it binds to and activates the NLRP3 inflammasome (41). It was also reported that ROS-mediated TXNIP detachment from thioredoxin triggered IL- $1 \beta$ expression (53). However, Masters et al. (30) identified no difference in NLRP3 inflammasome activation in response to inflammasome activators between WT and $\mathrm{TXNIP}^{-1-}$ macrophages, thereby excluding a role of TXNIP in modulating the NLRP3 inflammasome. Further studies need to be carried out to clarify the precise mechanism of ROS-mediated NLRP3 transcription.

However, there are studies indicating a negative regulatory role of ROS on NLRP3 inflammasome activation. It was found that excessive ROS could inhibit NLRP3-mediated caspase- 1 activation and that NLRP3 inflammasome activation and IL- $1 \beta$ secretion were suppressed by HO- 1 inhibitor zinc (II) protoporphyrin IX (ZnPP) and the knockdown of Nrf2 and with the consumption of GSH-induced superoxide anion production (15). Studies also showed that in SOD1deficient mice, persistent high ROS levels in macrophages were able to inhibit the activity of caspase- 1 and the maturation of IL-1 $\beta$ (31). ROS overproduction specifically inhibited caspase- 1 by reversible oxidation and glutathionylation of the redox-sensitive cysteine residues Cys397 and Cys362. A similar phenomenon may occur in $\mathrm{Nrf}^{-/-}$macrophages under chronic oxidative stress conditions, as Zhao suggested a positive role for Nrf2 in NLRP3 inflammasome activation (52). Further experiments are worth to dissect the precise mechanisms.

In conclusion, a model was proposed to illustrate how Nrf2 negatively regulated NLRP3 expression and subsequent caspase-1 activation (Fig. 10). NLRP3 is the rate-limiting element for the activation of the inflammasome. It has been demonstrated that the activation status of the NLRP3 inflammasome, which resulted from high level of NLRP3 expression, is closely related to many diseases, including metabolic diseases, autoinflammatory syndromes, and cancers. Hence, it is critical to tightly control NLRP3 expression to prevent and treat these diseases. Our work identified Nrf2 as an inhibitor of NLRP3 expression at the transcriptional level and provided a molecular pathway through an endogenous regulator affecting the immune response and even controlling the development of immune-mediated diseases. Furthermore, our results provided a strategy to downregulate the activity of inflammasome and suggested that Nrf 2 could be a potential target for the therapeutic modulation of NLRP3-associated diseases, such as autoinflammatory diseases, gout, and diabetes.

\section{Materials and Methods}

\section{Reagents}

tBHQ purchased from Sigma-Aldrich was dissolved in DMSO at $20 \mathrm{~m} M$. Primary antibodies for Nrf2, $\beta$-actin, lamin A, IL-1 $\beta$, horseradish peroxidase (HRP)-conjugated secondary antibodies were purchased from Bioworld Technology, Inc. NLRP3, NQO1, and caspase-1 antibody were purchased from CST Technology, Inc. Antibodies for flow cytometry, including CD11b, Ly-6C, and Ly-6G, were purchased from eBioscience (eBioscience, Inc.). Nrf2 siRNA and control siRNA were purchased from Santa Cruz Biotechnology, Inc. Phorbol myristate acetate (PMA), lipopolysaccharide (LPS), adenosine triphosphate (ATP), and rotenone were purchased from Sigma-Aldrich. Nacetyl-cysteine (NAC) and ROS Assay kit were purchased from Beyotime Institute of Biotechnology. ELISA kits for 
human and murine IL- $1 \beta$ were purchased from Boster Biotech Co. Ltd.

\section{Cell culture}

Human THP-1 cells were obtained from the Cell Bank of Shanghai, Institute of Biochemistry and Cell Biology, Chinese Academy of Sciences and maintained in RPMI 1640 medium (Gibco) supplemented with $10 \%$ heat-inactivated fetal bovine serum (Gibco) and $100 \mathrm{U} / \mathrm{ml}$ benzylpenicillin and $100 \mathrm{mg} / \mathrm{ml}$ streptomycin. THP-1 cells were stimulated by PMA $(100 \mathrm{ng} / \mathrm{ml})$ for $12 \mathrm{~h}$ to differentiate into macrophages. The peritoneal macrophages were isolated according to the following procedures. They were isolated from C57/BL6 mice and cultured with RPMI 1640 medium supplemented with $10 \%$ heat-inactivated fetal bovine serum. After $8 \mathrm{~h}$, culture fluid was exchanged with a fresh culture medium. The adherent macrophages were used for experiments. L929 cells were also purchased from the Institute of Biochemistry and Cell Biology and cultured with DMEM (Gibco) supplemented with $10 \%$ heat-inactivated fetal bovine serum. BMDM cells were isolated according to the following procedures. Bone marrow cells were isolated from C57/BL6 mice and cultured with DMEM supplemented with $10 \%$ heat-inactivated fetal bovine serum and 30\% L929 cellconditioned medium. Culture fluid was exchanged with a fresh culture medium every 3 days. Under these conditions, adherent macrophages were obtained within 7 days. Nonadherent cells were removed by washing the plate twice with PBS. The adherent macrophages were used for experiments. All cell lines were cultured under a humidified $5 \%(\mathrm{v} / \mathrm{v}) \mathrm{CO}_{2}$ atmosphere at $37^{\circ} \mathrm{C}$.

\section{Mice}

Six- to eight-week-old female C57BL/6 mice were purchased from Model Animal Genetics Research Center of Nanjing University (Nanjing, China). Six- to eight-week-old $\mathrm{Nrf}^{-1-}$ mice were on C57BL/6 background. Animal welfare and experimental procedures were carried out strictly in accordance with the Guide for the Care and Use of Laboratory Animals (National Institutes of Health, the United States) and the related ethical regulations of our university. All efforts were made to minimize animals' suffering and to reduce the number of animals used.

\section{Western blot assay}

Total proteins were extracted by adding RIPA lysis buffer with $1 \mathrm{~m} M$ PMSF for $1 \mathrm{~h}$ on the ice and centrifuging at $13,000 \mathrm{rpm}$ for $30 \mathrm{~min}$ at $4^{\circ} \mathrm{C}$. Protein concentration in the supernatants was measured by BCA protein assay (Thermo). Cell culture supernatants were concentrated with methanol/ chloroform and the pellets were lysed in the SDS sample buffer. Then, equal amount of sample was run on $12 \%$ SDSPAGE. The proteins were transferred to polyvinylidene difluoride (PVDF) membranes (Millipore) using a semidry transfer system (Bio-rad). Proteins were detected using specific antibodies of Nrf2, NQO1, NLRP3, caspase-1, $\beta$-actin, and lamin $\mathrm{A}$ overnight at $4^{\circ} \mathrm{C}$. The membranes were then washed three times with PBST followed by HRP-conjugated secondary antibodies for $1 \mathrm{~h}$ at $37^{\circ} \mathrm{C}$. All of the antibodies were diluted in PBST containing 1\% BSA. The signals were analyzed using the ECL chemiluminescence detection system (Tanon).

\section{Nuclear and cytoplasmic extraction}

Following treatments, cells were harvested by centrifugation and washed twice with PBS. Nuclear and cytosol lysates were isolated using a Nuclear/Cytosol Fractionation Kit (BioVision) according to the manufacturer's instruction. The protein concentration of the nuclear and cytoplasmic extracts was measured with the BCA protein assay reagent. Extracts were stored at $-20^{\circ} \mathrm{C}$ until further experimentation.

\section{Measurement of cytokine production}

Peritoneal macrophages and THP-1 cells were seeded in 12-well plates and cultured overnight. After different stimulation, the supernatants were collected, and the concentrations of IL- $1 \beta$ were measured using mouse IL- $1 \beta$ and human IL-1 $\beta$ ELISA kits (Boster) according to the manufacturer's instruction.

\section{RNA extraction and real-time RT-PCR}

Total RNA was isolated using the TRIzol reagent (Invitrogen) according to the manufacturer's protocol. The concentration and purity of the extracted RNA were measured with the optical densities at 260 and $280 \mathrm{~nm}$. RNA samples were reverse transcribed to cDNA and subjected to quantitative PCR, which was performed with the LightCycler $^{\circledR} 96$ Real-Time PCR System (Roche) using AceQ qPCR SYBR Green Master Mix (Vazyme). The primer sequences used in this study were as follows:

Nlrp3, 5'-AACATTCGGAGATTGTGGTTGGG-3' (forward)

5'-GTGCGTGAGATTCTGATTAGTGCTG-3' (reverse)

IL-1 $\beta, 5^{\prime}$-AGGCTGCTCTGGGATTC-3' (forward)

5'-GCCACAACAACTGACGC-3' (reverse)

GAPDH, 5'-AAGGTCGGAGTCAACGGATTT-3' (forward)

5'-AGATGATGACCCTTTTGGCTC-3' (reverse)

Nrf2, 5'-CGAGATATACGCAGGAGAGGTAAGA-3' (forward)

5'-GCTCGACAATGTTCTCCAGCTT-3' (reverse)

GCLC, 5' - GCTGTCTTGCAGGGAATGTT-3' (forward) 5'- ACACACCTTCCTTCCCATTG-3' (reverse)

GCLM， 5'- TTGGAGTTGCACAGCTGGATT-3' (forward)

5'- TGGTTTTACCTGTGCCCACTG-3' (reverse)

GST- CCTGTACCAGTCCAATACCATCCT-3' (forward)

5'- TCCTGCTGGTCCTTCCCATA-3' (reverse)

\section{Transfection of Nrf2 plasmid and Nrf2 siRNA}

Nrf2 plasmid was donated by Professor Siwang Yu from Peking University (Beijing, China) and Nrf2 siRNA was purchased from Santa Cruz Biotechnology, Inc. and transformed according to the manufacturer's instruction of Ecfect Transfection reagent (Vazyme).

\section{Measurement of intracellular ROS level}

ROS Assay kit purchased from Beyotime Institute of Biotechnology was used according to the manufacturer's 
instructions. Cells were cultured in a 12 -well plate, treated with various stimulations, and then, the cells were harvested and incubated with $100 \mu \mathrm{M}$ DCFH-DA attenuated with serum-free medium for $20 \mathrm{~min}$ at $37^{\circ} \mathrm{C}$ in the dark, washed twice with cold PBS. The fluorescence intensity was measured by the FACSCalibur flow cytometer (Becton Dickinson) at Ex./Em. $=488 / 525 \mathrm{~nm}$.

\section{Measurement of production of cellular superoxide}

THP-Ms superoxide levels were measured with dihydroethidium (DHE, Beyotime Institute of Biotechnology), tBHQ-pretreated THP-Ms suffered different stimulations, stained by DHE $\left(10 \mu \mathrm{M}, 30 \mathrm{~min}, 37^{\circ} \mathrm{C}\right)$ in the dark in a humidified chamber, briefly washed by PBS, and detected by FACS.

\section{Hydrogen peroxide assay}

The Hydrogen Peroxide Assay Kit was purchased from Beyotime Institute of Biotechnology. First, the cells were lysed by the schizolysis solution supplied by the kit. Then, the supernatants were collected by centrifuging at $1100 \mathrm{~g}$ for $10 \mathrm{~min}$ at $4^{\circ} \mathrm{C}$. Next, $50 \mu \mathrm{l}$ of supernatants and $100 \mu \mathrm{l}$ of test solutions were mixed and placed at room temperature for $20 \mathrm{~min}$ and measured instantly with a microplate reader at a wavelength of $570 \mathrm{~nm}$. Finally, the concentration of $\mathrm{H}_{2} \mathrm{O}_{2}$ released was calculated according to a standard concentration curve that originated from standard solutions on identical experiments.

\section{Measurement of SOD activity}

The SOD activity kit purchased was used according to the manufacturer's instructions. Cells were cultured in a six-well plate and treated with various stimulations. SOD activity was tested by measuring its inhibition on the process of xanthine oxidase catalyzing xanthine to generate superoxide anion free radicals, and then, the superoxide radicals could oxidize hydroxylamine to generate nitrite, which reacts with a developer to give a purple color. Based on this principle, we determine the SOD1 activity. The detailed procedures were performed as per the manufacturer's instruction.

\section{Measurement of catalase activity}

The Catalase Activity Kit purchased from Beyotime Institute of Biotechnology was used according to the manufacturer's instructions.

\section{Measurement of GSH}

The GSH kit purchased from Beyotime Institute of Biotechnology was used according to the manufacturer's instructions.

\section{Alum-induced peritonitis}

Mice were injected intraperitoneally with $700 \mu \mathrm{g}$ alum diluted in sterile PBS. Twelve hours later, peritoneal lavage was collected and PECs were stained with cell surface markers CD11b, Ly6C, and Ly6G. Flow cytometry analysis was performed by the FACSCalibur flow cytometer (Becton Dickinson).

\section{HE staining}

Peritoneal macrophages were seeded in six-well plates and cultured for $8 \mathrm{~h}$, and the standard HE staining was performed according to the manufacturer's instructions. Hematoxylin was used to stain nucleic acids and eosin was used to stain the cytoplasm and extracellular matrix.

\section{Statistical analyses}

Results are presented as the mean \pm SD. Statistical analyses were performed with the $t$-test for two groups or one-way ANOVA (GraphPad Software) for multiple groups. $p$ Values $<0.05$ were considered significant.

\section{Acknowledgments}

This work was supported by the National Natural Science Foundation of China (No. 81372268, 81173087, and 81202611), Program of State Key Laboratory of Natural Medicines, China Pharmaceutical University (No.SKLNMZZCX201405), Natural Science Foundation for Distinguished Young Scholars of Jiangsu Province (No. BK20130026), and the Program for Jiangsu Province Innovative Research Team. We want to thank Professor Siwang $\mathrm{Yu}$ from Peking University for the $\mathrm{Nrf}^{-1-}$ mice.

\section{Author Disclosure Statement}

The authors state no conflicts of interest exist.

\section{References}

1. Bauernfeind F, Bartok E, Rieger A, Franchi L, Núñez G, and Hornung V. Cutting edge: reactive oxygen species inhibitors block priming, but not activation, of the NLRP3 inflammasome. J Immunol 187: 613-617, 2011.

2. This reference has been deleted.

3. Bauernfeind FG, Horvath G, Stutz A, Alnemri ES, MacDonald K, Speert D, Fernandes-Alnemri T, Wu J, Monks BG, Fitzgerald KA, Hornung V, and Latz E. Cutting edge: NF-kappaB activating pattern recognition and cytokine receptors license NLRP3 inflammasome activation by regulating NLRP3 expression. J Immunol 183: 787-791, 2009.

4. Bellingan GJ, Xu P, Cooksley H, Cauldwell H, Shock A, Bottoms S, Haslett C, Mutsaers SE, and Laurent GJ. Adhesion molecule-dependent mechanisms regulate the rate of macrophage clearance during the resolution of peritoneal inflammation. J Exp Med 196: 1515-1521, 2002.

5. Davis BK, Wen H, and Ting JP. The inflammasome NLRs in immunity, inflammation, and associated diseases. Ann Rev Immunol 29: 707, 2011.

6. Dowling JK and O'Neill LA. Biochemical regulation of the inflammasome. Crit Rev Biochem Mol Biol 47: 424-443, 2012.

7. Duan X, Li J, Li W, Xing X, Zhang Y, Li W, Zhao L, Sun $\mathrm{G}, \mathrm{Gao} \mathrm{XH}$, and Li B. Antioxidant tert-butylhydroquinone ameliorates arsenic-induced intracellular damages and apoptosis through induction of Nrf2-dependent antioxidant responses as well as stabilization of anti-apoptotic factor Bcl-2 in human keratinocytes. Free Radic Biol Med 94: 7487, 2016.

8. Fukatsu K, Saito H, Han I, Yasuhara H, Lin MT, Inoue T, Furukawa S, Inaba T, Hashiguchi Y, Matsuda T, and Muto $\mathrm{T}$. The greater omentum is the primary site of neutrophil 
exudation in peritonitis. $J$ Am Coll Surg 183: 450-456, 1996.

9. Guarda G, Braun M, Staehli F, Tardivel A, Mattmann C, Forster I, Farlik M, Decker T, Du Pasquier RA, Romero P, and Tschopp J. Type I interferon inhibits interleukin-1 production and inflammasome activation. Immunity 34: 213-223, 2011.

10. Hewinson J, Moore SF, Glover C, Watts AG, and MacKenzie $\mathrm{AB}$. A key role for redox signaling in rapid $\mathrm{P} 2 \mathrm{X} 7$ receptor-induced IL-1 beta processing in human monocytes. J Immunol 180: 8410-8420, 2008.

11. Higgins LG, Kelleher MO, Eggleston IM, Itoh K, Yamamoto M, and Hayes JD. Transcription factor Nrf2 mediates an adaptive response to sulforaphane that protects fibroblasts in vitro against the cytotoxic effects of electrophiles, peroxides and redox-cycling agents. Toxicol Appl Pharmacol 237: 267-280, 2009.

12. Hu R, Saw CL-L, Yu R, and Kong A-NT. Regulation of NF-E2-related factor 2 signaling for cancer chemoprevention: antioxidant coupled with antiinflammatory. Antioxid Redox Signal 13: 1679-1698, 2010.

13. Huai W, Zhao R, Song H, Zhao J, Zhang L, Zhang L, Gao C, Han L, and Zhao W. Aryl hydrocarbon receptor negatively regulates NLRP3 inflammasome activity by inhibiting NLRP3 transcription. Nat Commun 5: 4738, 2014.

14. Jankovic D, Ganesan J, Bscheider M, Stickel N, Weber FC, Guarda G, Follo M, Pfeifer D, Tardivel A, and Ludigs K. The Nlrp3 inflammasome regulates acute graft-versus-host disease. J Exp Med 210: 1899-1910, 2013.

15. Jhang J-J, Cheng Y-T, Ho C-Y, and Yen G-C. Monosodium urate crystals trigger Nrf2-and heme oxygenase-1dependent inflammation in THP-1 cells. Cell Mol Immunol 12: 424-434, 2014.

16. Jin J, Yu Q, Han C, Hu X, Xu S, Wang Q, Wang J, Li N, and Cao X. LRRFIP2 negatively regulates NLRP3 inflammasome activation in macrophages by promoting Flightless-I-mediated caspase-1 inhibition. Nat Commun 4: 2075, 2013.

17. Juliana C, Fernandes-Alnemri T, Kang S, Farias A, Qin F, and Alnemri ES. Non-transcriptional priming and deubiquitination regulate NLRP3 inflammasome activation. $J$ Biol Chem 287: 36617-36622, 2012.

18. Ka SM, Lin JC, Lin TJ, Liu FC, Chao LK, Ho CL, Yeh LT, Sytwu HK, Hua KF, and Chen A. Citral alleviates an accelerated and severe lupus nephritis model by inhibiting the activation signal of NLRP3 inflammasome and enhancing Nrf2 activation. Arthritis Res Ther 17: 331, 2015.

19. Khor TO, Huang M-T, Prawan A, Liu Y, Hao X, Yu S, Cheung WKL, Chan JY, Reddy BS, and Yang CS. Increased susceptibility of Nrf2 knockout mice to colitis-associated colorectal cancer. Cancer Prev Res 1: 187-191, 2008.

20. Kim J, Cha Y-N, and Surh Y-J. A protective role of nuclear factor-erythroid 2-related factor-2 (Nrf2) in inflammatory disorders. Mutat Res 690: 12-23, 2010.

21. Kim TW, Kim YJ, Kim HT, Park SR, Lee MY, Park YD, Lee $\mathrm{CH}$, and Jung JY. NQO1 deficiency leads enhanced autophagy in cisplatin-induced acute kidney injury through the AMPK/TSC2/mTOR signaling pathway. Antioxid Redox Signal 24: 867-883, 2016.

22. Kolb R, Liu G-H, Janowski AM, Sutterwala FS, and Zhang W. Inflammasomes in cancer: a double-edged sword. Protein Cell 5: 12-20, 2014.

23. Kovac S, Angelova PR, Holmström KM, Zhang Y, Dinkova-Kostova AT, and Abramov AY. Nrf2 regulates
ROS production by mitochondria and NADPH oxidase. Biochim Biophys Acta 1850: 794-801, 2015.

24. Lamkanfi M and Dixit VM. Inflammasomes and their roles in health and disease. Annu Rev Cell Dev Biol 28: 137-161, 2012.

25. Latz E, Xiao TS, and Stutz A. Activation and regulation of the inflammasomes. Nat Rev Immunol 13: 397-411, 2013.

26. Leong PK and Ko KM. Schisandrin B induces an Nrf2mediated thioredoxin expression and suppresses the activation of inflammasome in vitro and in vivo. Biofactors 41: 314-323, 2015.

27. Li B, Abdalrahman A, Lai Y, Janicki JS, Ward KW, Meyer CJ, Wang XL, Tang D, and Cui T. Dihydro-CDDOtrifluoroethyl amide suppresses inflammatory responses in macrophages via activation of Nrf2. Biochem Biophys Res Commun 444: 555-561, 2014.

28. Li W, Khor TO, Xu C, Shen G, Jeong W-S, Yu S, and Kong A-N. Activation of Nrf2-antioxidant signaling attenuates $\mathrm{NF} \kappa \mathrm{B}$-inflammatory response and elicits apoptosis. Biochem Pharmacol 76: 1485-1489, 2008.

29. Lin K-M, Hu W, Troutman TD, Jennings M, Brewer T, Li X, Nanda S, Cohen P, Thomas JA, and Pasare C. IRAK-1 bypasses priming and directly links TLRs to rapid NLRP3 inflammasome activation. Proc Natl Acad Sci USA 111: 775-780, 2014.

30. Masters SL, Dunne A, Subramanian SL, Hull RL, Tannahill GM, Sharp FA, Becker C, Franchi L, Yoshihara E, Chen Z, Mullooly N, Mielke LA, Harris J, Coll RC, Mills KH, Mok KH, Newsholme P, Nunez G, Yodoi J, Kahn SE, Lavelle EC, and O'Neill LA. Activation of the NLRP3 inflammasome by islet amyloid polypeptide provides a mechanism for enhanced IL-1beta in type 2 diabetes. Nat Immunol 11: 897-904, 2010.

31. Meissner F, Molawi K, and Zychlinsky A. Superoxide dismutase 1 regulates caspase-1 and endotoxic shock. Nat Immunol 9: 866-872, 2008.

32. Pan CW, Pan ZZ, Hu JJ, Chen WL, Zhou GY, Lin W, Jin $\mathrm{LX}$, and $\mathrm{Xu} \mathrm{CL}$. Mangiferin alleviates lipopolysaccharide and D-galactosamine-induced acute liver injury by activating the Nrf2 pathway and inhibiting NLRP3 inflammasome activation. Eur J Pharmacol 770: 85-91, 2016.

33. Pang C, Zheng Z, Shi L, Sheng Y, Wei H, Wang Z, and Ji L. Caffeic acid prevents acetaminophen-induced liver injury by activating the Keap1-Nrf2 antioxidative defense system. Free Radic Biol Med 91: 236-246, 2016.

34. Rangasamy T, Guo J, Mitzner WA, Roman J, Singh A, Fryer AD, Yamamoto M, Kensler TW, Tuder RM, and Georas SN. Disruption of Nrf2 enhances susceptibility to severe airway inflammation and asthma in mice. J Exp Med 202: 47-59, 2005.

35. Rubartelli A. Redox control of NLRP3 inflammasome activation in health and disease. J Leukoc Biol 92: 951-958, 2012.

36. This reference has been deleted.

37. Satoh T, Kambe N, and Matsue H. NLRP3 activation induces ASC-dependent programmed necrotic cell death, which leads to neutrophilic inflammation. Cell Death Dis 4: e644, 2013.

38. Schroder K and Tschopp J. The inflammasomes. Cell 140: 821-832, 2010.

39. Shaw PJ, McDermott MF, and Kanneganti TD. Inflammasomes and autoimmunity. Trends Mol Med 17: 57-64, 2011.

40. Shimada K, Crother TR, Karlin J, Dagvadorj J, Chiba N, Chen S, Ramanujan VK, Wolf AJ, Vergnes L, Ojcius DM, Rentsendorj A, Vargas M, Guerrero C, Wang Y, Fitzgerald KA, Underhill DM, Town T, and Arditi M. Oxidized mi- 
tochondrial DNA activates the NLRP3 inflammasome during apoptosis. Immunity 36: 401-414, 2012.

41. This reference has been deleted.

42. Suzuki T, Motohashi H, and Yamamoto M. Toward clinical application of the Keap1-Nrf2 pathway. Trends Pharmacol Sci 34: 340-346, 2013.

43. Thimmulappa RK. Nrf2 is a critical regulator of the innate immune response and survival during experimental sepsis. J Clin Invest 116: 984-995, 2006.

44. Thimmulappa RK, Lee H, Rangasamy T, Reddy SP, Yamamoto M, Kensler TW, and Biswal S. Nrf2 is a critical regulator of the innate immune response and survival during experimental sepsis. J Clin Invest 116: 984, 2006.

45. Turley AE, Zagorski JW, and Rockwell CE. The Nrf2 activator tBHQ inhibits $\mathrm{T}$ cell activation of primary human CD4 T cells. Cytokine 71: 289-295, 2015.

46. Van de Veerdonk FL, Netea MG, Dinarello CA, and Joosten LA. Inflammasome activation and IL- $1 \beta$ and IL-18 processing during infection. Trends Immunol 32: 110-116, 2011.

47. Wagner AE, Will O, Sturm C, Lipinski S, Rosenstiel P, and Rimbach G. DSS-induced acute colitis in C57BL/6 mice is mitigated by sulforaphane pre-treatment. J Nutr Biochem 24: 2085-2091, 2013.

48. This reference has been deleted.

49. Wang Y, Wang H, Qian C, Tang J, Zhou W, Liu X, You Q, and $\mathrm{Hu}$ R. 3-(2-Oxo-2-phenylethylidene)-2,3,6,7-tetrahydro1H-pyrazino[2,1-a]isoquinolin-4(1 1bH)-one (compound 1), a novel potent Nrf2/ARE inducer, protects against DSSinduced colitis via inhibiting NLRP3 inflammasome. Biochem Pharmacol 101: 71-86, 2016.

50. Yang SM, Ka SM, Hua KF, Wu TH, Chuang YP, Lin YW, Yang FL, Wu SH, Yang SS, Lin SH, Chang JM, and Chen A. Antroquinonol mitigates an accelerated and progressive IgA nephropathy model in mice by activating the Nrf2 pathway and inhibiting T cells and NLRP3 inflammasome. Free Radic Biol Med 61: 285-297, 2013.

51. Zhao C, Gillette DD, Li X, Zhang Z, and Wen H. Nuclear factor E2-related factor-2 (Nrf2) is required for NLRP3 and AIM2 inflammasome activation. J Biol Chem 289: 1702017029, 2014.

52. This reference has been deleted.

53. Zhou R, Tardivel A, Thorens B, Choi I, and Tschopp J. Thioredoxin-interacting protein links oxidative stress to inflammasome activation. Nat Immunol 11: 136-140, 2010.
54. Zhou R, Yazdi AS, Menu P, and Tschopp J. A role for mitochondria in NLRP3 inflammasome activation. Nature 469: 221-225, 2011.

$$
\begin{array}{r}
\text { Address correspondence to: } \\
\text { Prof. Rong Hu } \\
\text { Department of Physiology } \\
\text { State Key Laboratory of Natural Medicines } \\
\text { China Pharmaceutical University } \\
24 \text { Tongjia Xiang } \\
\text { Nanjing 210009 } \\
\text { China } \\
\text { E-mail: ronghu@cpu.edu.cn }
\end{array}
$$

Date of first submission to ARS Central, December 31, 2015; date of final revised submission, June 12, 2016; date of acceptance, June 14, 2016.

\section{Abbreviations Used}

$\mathrm{ARE}=$ antioxidant response element

$\mathrm{ASC}=$ apoptosis-associated speck-like protein that contains a CARD

$\mathrm{ATP}=$ adenosine triphosphate

$\mathrm{BMDM}=$ bone marrow-derived macrophage

$\mathrm{GSH}=$ glutathione

IL-18 = interleukin-18

IL-1 $\beta=$ interleukin- $1 \beta$

LPS $=$ lipopolysaccharide

$\mathrm{NAC}=\mathrm{N}$-acetyl-cysteine

$\mathrm{NF}-\kappa \mathrm{B}=$ nuclear factor $-\kappa \mathrm{B}$

NLR $=$ NOD-like receptor

NLRP3 = NLR family, pyrin domain containing 3

$\mathrm{Nrf} 2=$ nuclear factor erythroid 2-related factor 2

$\mathrm{PEC}=$ peritoneal exudate cell

PMA $=$ phorbol myristate acetate

ROS $=$ reactive oxygen species

$\mathrm{SOD}=$ superoxide dismutase

tBHQ $=$ tert-butylhydroquinone

THP-Ms $=$ THP- 1 cell-derived macrophages

TNF- $\alpha=$ tumor necrosis factor $\alpha$ 